\title{
Total Synthesis of the Amaryllidaceae Alkaloid Clivonine
}

\author{
Helmut Haning, ${ }^{a, b}$ Carles Giró Mañas, ${ }^{c}$ Victoria L. Paddock, ${ }^{c}$ Christian G. Bochet, ${ }^{d, b}$ Andrew J. P. White, ${ }^{c}$ \\ Gerald Bernardinelli, ${ }^{b}$ Inderjit Mann, ${ }^{e}$ Wolfang Oppolzer, ${ }^{\dagger b}$ and Alan C. Spivey ${ }^{* c, b}$
}

\author{
Receipt/Acceptance Data [DO NOT ALTER/DELETE THIS TEXT] \\ ${ }_{5}$ Publication data [DO NOT ALTER/DELETE THIS TEXT] \\ DOI: 10.1039/b000000x [DO NOT ALTER/DELETE THIS TEXT]
}

Two syntheses of the Amaryllidaceae alkaloid clivonine (1) are described. Both employ previously reported 7-arylhydrindane $\mathbf{6}$ as an intermediate but differ in the method employed for subsequent introduction of what becomes the ring-B lactone carbonyl carbon (C7). The synthesis featuring a

${ }_{10}$ Bischler-Napieralski reaction for this transformation constitutes the first asymmetric synthesis of natural (+)-clivonine. Crystal structures for compounds ( \pm -13, $( \pm)-\mathbf{1 6},(-)-20$ and $( \pm)-\mathbf{2 8}$ are also reported.

\section{Introduction}

The Amaryllidaceae are herbaceous perennials that produce a 15 diverse array of alkaloid secondary metabolites. These alkaloids can mostly be classified as belonging to one of eight skeletally distinct subclasses all derived biosynthetically from a common precursor, norbelladine. ${ }^{1-3}$ Clivonine (1), along with homolycorine (2), hippeastrine (3) and lycorenine (4) are 20 prominent members of the lycorenine subclass; these alkaloids feature a tetracyclic 2-benzopyrano-[3,4-g]indole skeleton and display growth inhibition of various tumour cells, ${ }^{4-6}$ DNA binding properties, ${ }^{7}$ anti-viral activity, ${ }^{8,}{ }^{9}$ antifungal activity, ${ }^{10}$ and insect antifeedant activity ${ }^{11}$ (Figure 1). ${ }^{12}$
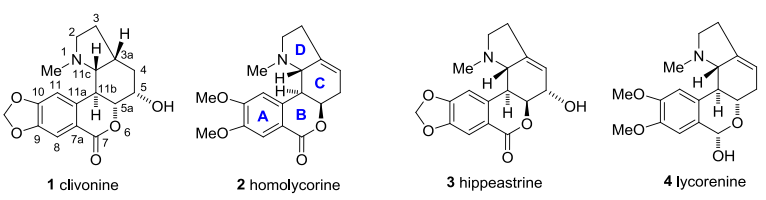

Figure 1. Structures of the lycorenine-class Amaryllidaceae alkaloids clivonine (1), homolycorine (2), hippeastrine (3) and lycorenine (4). Also showing atom numbering/ring designations and correct absolute stereochemistry for natural (+)-clivonine (i.e. $3 \mathrm{a} R, 5 S, 5 \mathrm{a} R, 11 \mathrm{~b} S, 11 \mathrm{c} R$ ).

(+)-Clivonine (1) was first isolated as white prisms in $0.0007 \%$ yield from an ethanolic extract of freshly collected rhizomes and leaves of Clivia miniata Regel in 1956 by Wildman, who also tentatively proposed the correct gross

\footnotetext{
${ }^{a}$ Bayer Schering Pharma AG, Müllerstr. 178, 13353 Berlin, Germany.

${ }^{b}$ Department of Organic Chemistry, University of Geneva, 30, quai

Ernest Ansermet, CH-1211 Geneva 4, Switzeland.

${ }^{c}$ Department of Chemistry, Imperial College London, South Kensington campus, London, SW7 2AZ, UK Fax: (+) 44 (0)20 75945841; Tel: (+) 44 (0)20 75945841; E-mail: a.c.spivey@imperial.ac.uk

${ }^{d}$ Department of Chemistry, University of Fribourg, $9 \mathrm{Ch}$. du Musee, CH1700 Fribourg, Switzerland.

${ }^{e}$ GSK Tonbridge, Old Powder Mills, Leigh, Tonbridge, Kent, TN11 9AN $U K$.

Electronic Supplementary Information (ESI) available: crystallographic analyses for compounds $( \pm)-\mathbf{1 3},( \pm)-\mathbf{1 6},(-)-20$ and $( \pm)-28$ including CIF files. Details of the DFT modelling for compound $\mathbf{1 1}$ and its C3a epimer and NMR spectra for compounds 1, 8, 9, 11-13, 16 and 25-31. See http://dx.doi.org/10.1039/b000000x/
}

topology of the molecule. ${ }^{13}$ In 1965 Mehlis suggested relative 30 (and absolute) stereochemistry for (+)-clivonine but assigned the stereochemistry of the $\mathrm{C} 5 / 5 \mathrm{a}$ cis-diol centres relative to the others incorrectly. ${ }^{14}$ In 1967 the stereochemical assignment of these centres was revised by Döpke et al. from ${ }^{1} \mathrm{H}$ NMR data of the C5 acetate derivative ${ }^{15}$ and in 1971 these assignments and the 35 absolute stereochemistry were confirmed by Jeffs et al. via chemical correlation with $\alpha$-dihydrohippeastrine ${ }^{\ddagger 16-19}$

Irie developed the first total synthesis of $( \pm)$-clivonine in $1973^{20-22}$ (17 steps, $0.43 \%$ overall yield from piperonal) and we have recently described the second ${ }^{23}$ (12 steps, $6.1 \%$ overall yield 40 from enone 5). Our synthesis featured the use of a thermal retroCope elimination reaction ${ }^{24}$ to install the B-C and C-D ringjunction stereochemistry in a 7-arylhydrindane advanced intermediate $\mathbf{6}^{25}$ This compound was then converted into a lycorine-type iminium salt having a $1 H$-pyrrolo[3,2,1$45 \mathrm{~d}$,e]phenanthridine/galanthan skeleton, which by sequential hydration, methylation and oxidation underwent a biomimetic 'ring-switch' to give clivonine (1) (Scheme 1).

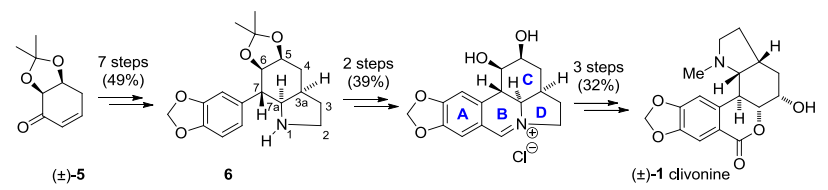

Scheme 1. Our previous synthesis of $( \pm)$-clivonine (1) from enone $( \pm)-5$ (see ref. $^{23}$ ). Also showing atom numbering for 7 -arylhydrindane (octahydroindole) 6 and ring designations for the subsequent lycorinetype (galanthan) salt.

Herein, we describe two alternative syntheses of clivonine (1), from 7-arylhydrindane 6, one via a lycorine-type intermediate 50 but featuring a non-biomimetic sequence of reactions to effect the 'ring-switch' and the other not proceeding via a lycorine-type intermediate. These syntheses were primarily developed to deliver authentic samples of clivonine (1) in order to aid development of the biomimetic 'ring-switch' approach and have 55 therefore not been fully optimised. The first of these syntheses was carried out from enantiomerically pure enone (+)-5 and therefore constitutes the first asymmetric synthesis of natural (+)clivonine (1). 


\section{Results and Discussion}

60 For the synthesis of enone $( \pm)-5$ two routes were used both starting from meso-cyclohexa-3,5-dienyl-1,2-diol acetonide. ${ }^{\$ 26-29}$ The first was via a telescoped variant ${ }^{23}$ of the method described by Hudlicky ${ }^{30}$ and by Borchardt ${ }^{31}$ involving epoxidation then $\operatorname{Pd}(0) /$ acid-mediated rearrangement, and the second was via ${ }_{65}$ singlet oxygen addition $^{27}$ then Kornblum-DeLaMare rearrangement. ${ }^{32,33}$ Both afforded similar overall yields of enone $( \pm)-5$ but the former was preferred on step-count and reaction duration grounds. Synthesis of enone $(+)-(5 S, 6 S)-5$ was from commercially available Pseudomonas putida derived (+)-(2S,3S)70 1-chloro-4,6-cyclohexadiene-2,3-diol acetonide via a modification $^{34}$ of the method described by Oppolzer ${ }^{35}$ involving singlet oxygen addition, polystyrene-supported thiourea mediated hydrogenolysis/acetate protection then $\operatorname{Pd}(0) / \mathrm{TMDS}$ mediated reduction.

75 These enones were converted through to 7-arylhydrindanes ( \pm )-6 and (+)-6 according to our published procedure; ${ }^{23}$ the following summary draws attention to some synthetic details that were not previously highlighted for this sequence of steps (Scheme 2).

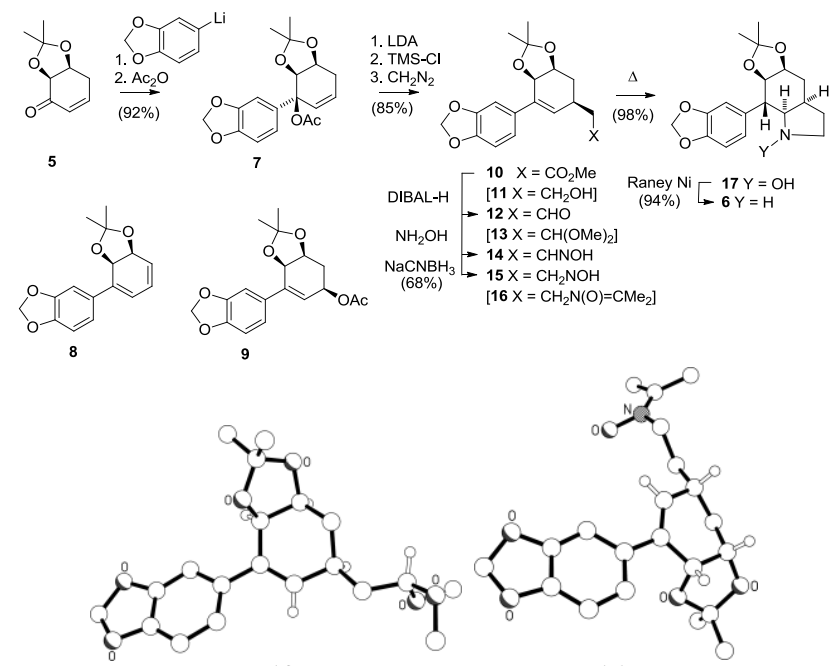

$( \pm)-13$

$( \pm)-16$

Scheme 2. Synthesis of 7-arylhydrindane 6 and the molecular structures of acetal $( \pm)-\mathbf{1 3}$ and nitrone ( \pm )-16 (X-ray).

80 1,2-Aryl lithium addition and acetate trapping $(\mathbf{5} \rightarrow \mathbf{7}, 92 \%$ yield) requires $2 \mathrm{~h}$ at $-78{ }^{\circ} \mathrm{C}$ in $\mathrm{THF}$, followed by warming to RT, and then addition of freshly distilled acetic anhydride. Development of the Ireland-Claisen rearrangement $(\mathbf{7} \rightarrow \mathbf{1 0})$ required some optimisation as it was found that use of $n$-BuLi as 85 a base led to exclusive formation of diene $\mathbf{8}$ (95\% yield) and use of too dilute solutions of LDA led simply to rearrangement to the 1,3-transposed allylic acetate 9 (43\% yield). However, the optimised Ireland-Claisen conditions could even be performed on the crude acetate 7 and the resulting acid reacted directly with 90 diazomethane following workup to give the methyl ester $\mathbf{1 0}$ on a large scale $(\sim 20 \mathrm{~g})$ in yields of $65-85 \%$ over the 3 steps. Conversion of this ester to the aldehyde 12 was carried out either directly using DIBAL-H at low temperature or by reduction to alcohol 11 using $\mathrm{LiBH}_{4}$ then re-oxidation using the Dess-Martin
95 periodinane; yields were comparable (80-85\%). Interestingly, attempted conversion of aldehyde $\mathbf{1 2}$ into the corresponding oxime 14 using hydroxylamine hydrochloride and magnesium sulfate in $\mathrm{MeOH}$ let to formation of the dimethylacetal $\mathbf{1 3}$ (39\% yield) on which a single crystal X-ray structure determination 100 was performed to confirm the relative stereochemistry in the racemic series. Omission of the dehydrating agent and use of thermally milder conditions however gave the oxime $\mathbf{1 4}$ as a $\sim 1.2: 1$ mixture of $(E)$ - and $(Z)$-isomers in $82 \%$ yield. The Borch reduction of this mixture using $\mathrm{NaCNBH}_{3} / \mathrm{HCl}$ required precise 105 control of the dilution and $\mathrm{pH}$ (using methyl orange) of the reaction medium and the duration of the reaction. A range of unidentified products formed when $\mathrm{HCl}$ addition was too slow (i.e. $\mathrm{pH}>4$ ) and nitrone 16, the structure of which was secured by a single crystal X-ray structure determination, was the 110 unexpected major product when $\mathrm{HCl}$ addition was too fast (i.e. $\mathrm{pH}<3$ ). This product presumably arises from partial deprotection of the acetonide under the acidic conditions and condensation of the released acetone with hydroxylamine 15 (the yield never exceeded 48\%). However, under optimised conditions the labile 115 hydroxylamine $\mathbf{1 5}$ could be obtained in $83 \%$ yield. Direct thermolysis of this compound in degassed toluene effected the retro-Cope elimination reaction to give the $N$-hydroxy-7arylhydrindane 17 almost quantitatively (98\% yield). Relatively high dilution is important in this reaction to preclude competing 120 disproportionation to the corresponding amine and nitro derivatives and degassing is required to prevent radical coupling to an azoxydimer. Raney-Ni hydrogenolysis of $\mathrm{N}$-hydroxy-7arylhydrindane $\mathbf{6}$ in wet ethanol gave the 7-arylhydrindane $\mathbf{6}$ in $94 \%$ yield.

125 A particular aspect of the above synthesis that intrigued us was the fact that the retro-Cope elimination reaction of hydroxylamine $\mathbf{1 5}$ proceeded significantly more rapidly than that of its C3a epimer, which we had previously studied en route to lycorine-type alkaloid (+)-trianthine, when compared under 130 identical conditions $($ i.e. $\mathbf{1 5} \rightarrow \mathbf{1 7}, 91 \%$ yield after 14 h vs. $93 \%$ after $70 \mathrm{~h}$ for its $\mathrm{C} 3 \mathrm{a}$ epimer, both in degassed benzene at 0.01 M). ${ }^{35}$ Inspection of the ${ }^{1} \mathrm{H}$ NMR spectra of pre-cyclisation intermediates in both series (i.e. compounds 10, 11, 12, 14 and $\mathbf{1 5}^{23} c f$. their $\mathrm{C} 3 \mathrm{a}$ epimers ${ }^{35}$ ) reveals that the protons in their 135 respective cyclohexene C-rings display systematic differences indicating that the two series adopt conformationally distinct halfchair structures in solution.** However, the relative positions of $\mathrm{H} 3 \mathrm{a}$ suggest that in the clivonine series this proton is pseudoaxial (and hence the C3a side chain pseudo-equatorial) and in the 140 trianthine series it is pseudo-equatorial (and the C3a side-chain pseudo-axial) indicating that both their fused-bicyclic (dioxolane/cyclohexene) cores adopt conformations in which the allylic C6a-O bond is pseudo-axial (e.g. for alcohol $11 \mathrm{H} 3 \mathrm{a}$ is at $\delta$ $2.45 \mathrm{ppm} c f$. its epimer at $\delta 2.63 \mathrm{ppm})^{36}$. These conformational 145 preferences were corroborated by DFT molecular modelling at the $6-31 G(d, p)$ level in the gas phase. The crystal structures of compounds 13 and 16 (Scheme 2) reveal that, at least in the clivonine series, this conformation persists also in the solid phase. Since retro-Cope elimination is geometrically precluded unless 150 the $\mathrm{C} 3$ a side chain adopts a pseudo-axial conformation this means that the faster cyclising epimer $\mathbf{1 5}$, leading to clivonine, must ring-flip in order to react. This places the aforementioned allylic 
$\mathrm{C}-\mathrm{O}$ bond pseudo-equatorial and the corresponding allylic $\mathrm{C}-\mathrm{H}$ bond pseudo-axial. Strain-release, or a favourable H-bonding 155 interaction between the hydroxylamine hydroxyl and one of the syn dioxolane oxygens in the transition state ${ }^{24}$ might explain the higher rate of cyclisation but it likely reflects more facile cyclisation on the alkene face anti to the pseudo-axial allylic $\sigma_{\mathrm{C}-\mathrm{H}}$ bond (i.e. the best donor, $c f$. a Cieplak effect, Scheme 3$)^{37-39+\dagger}$.

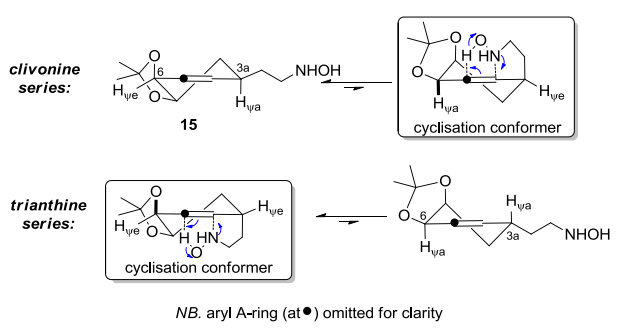

Scheme 3. Conformational analysis of the retro-Cope elimination substrate 15 (clivonine series) and its C3a epimer (trianthine series) ${ }^{35}$ showing the relative orientations of the $\mathrm{CH}$ and $\mathrm{CO}$ bonds at their respective allylic C6 stereocentres. A favourable $\sigma_{\mathrm{CH}} \rightarrow \pi^{*}{ }_{\mathrm{CC}}$ Cieplak stereoelectronic interaction ( $\left.c f . \sigma_{\mathrm{CH}} \rightarrow \pi^{*}{ }_{\mathrm{CC}}\right)$ may account for the more facile cyclisation in the clivonine series.

160 Based on precedent from the work of Mizukami, ${ }^{42}$ Kotera $e t$ al. ${ }^{43}$ and Harken et $a l .{ }^{44}$ who have reported multi-step conversions of isolated natural lycorine-type alkaloids to lycorenine-type congeners exploiting the classical von Braun reaction $^{45}$ (using cyanogen bromide), we envisaged that a related 165 strategy could be deployed to access clivonine (1) from lycorinetype progenitor 20. The synthesis of $1 H$-pyrrolo[3,2,1d,e]phenanthridine 20 from 7-arylhydrindane $\mathbf{6}$ was envisaged via a Pictet-Spengler reaction to form ring-B based on our successful use of this approach in the synthesis (+)-trianthine from a 170 diastereomeric 7-arylhydrindane ${ }^{35}$ (Scheme 4).
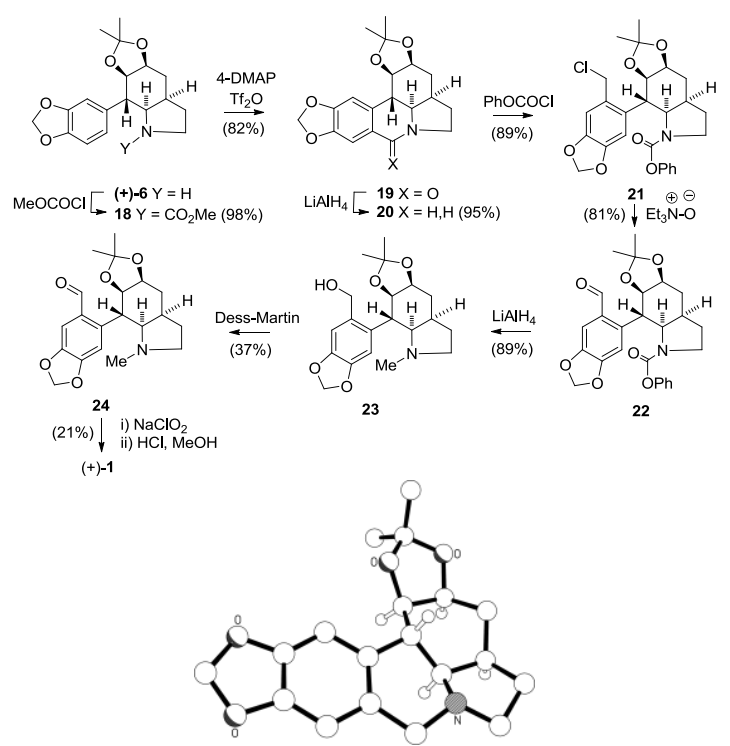

(-)-20

Scheme 4. Synthesis of clivonine (+)-1 using a von Braun-type reaction as a key step and the molecular structure of amine (-)-20 (X-ray).
In the event, direct Pictet-Spengler ring closure to give amine 20 using Eschenmoser's salt ( $N, N$-dimethyliminium iodide $)^{46,47}$ proved to be less high yielding than in the trianthine series $(53 \%$ cf. $92 \%{ }^{35}$ ). Since this yield could not be improved by the use of 175 the corresponding $N, N$-dimethyliminium triflate, ${ }^{48}$ we employed a three-step approach via methyl carbamate formation $(\rightarrow \mathbf{1 8}, 98 \%$ yield), a Bischler-Napieralski reaction using Banwell's conditions $^{49,50}$ (4-DMAP/Tf $2 \mathrm{O}, \rightarrow \mathbf{1 9}, 82 \%$ yield) and then lactam reduction $\left(\mathrm{LiAlH}_{4}, \rightarrow \mathbf{2 0}, 95 \%\right.$ yield $){ }^{\star}$ A single crystal X-ray 180 structure determination on amine $\mathbf{2 0}$ confirmed the introduction of the desired trans B-C and cis C-D ring-junction stereochemistry by the retro-Cope elimination reaction and the successful completion of the required galanthan skeleton (Scheme 4). The stage was now set for the von Braun-type 185 cleavage of the benzylic $\mathrm{C}-\mathrm{N}$ bond in ring-B. The optimum reagent for this key step was found to be phenylchloroformate, ${ }^{51}$ which proved superior to both ethylchloroformate ${ }^{52}$ and $\alpha$ chloroethylchloroformate $^{53}$ and gave the ring-opened benzyl chloride 21 in $89 \%$ yield when used in conjunction with $\mathrm{NaHCO}_{3}$ 190 in refluxing $\mathrm{CH}_{2} \mathrm{Cl}_{2}$. Conversion of this chloride to an oxygenbased function turned out to be challenging: attempted silver-salt assisted nucleophilic substitution resulted in reclosure of ring-B to give amine $\mathbf{2 0}$ and treatment with KOAc/TMEDA, ${ }^{54}$ polymersupported carbonate, ${ }^{55}$ and $\mathrm{CrO}_{3},{ }^{56}$ gave multiple unidentified 195 products. Finally, introduction of oxygen at the benzylic position could be achieved by oxidation with trimethylamine- $N$-oxide ${ }^{57}$ giving aldehyde $\mathbf{2 2}$ in $81 \%$ yield. Treatment of this compound with $\mathrm{LiAlH}_{4}$ effected conversion of the carbamate to the desired $\mathrm{N}$-methyl function albeit with concomitant aldehyde to alcohol 200 reduction giving aminoalcohol 23 in $89 \%$ yield. A reversed order of events was also explored but all attempts at carbamate reduction directly on benzyl chloride $\mathbf{2 1}$ using a variety of hydride reagents (e.g. $\mathrm{LiAlH}_{4}$ ) effected reclosure of ring-B to give amine 20 exclusively. Reoxidation of alcohol 23 could not 205 be realized via various Swern-type protocols ${ }^{58}$ but conversion to aldehyde $\mathbf{2 4}$ was achieved, albeit in a disappointing yield of $37 \%$ using the Dess-Martin periodinane ${ }^{59}{ }^{\S \S}$ Finally, treatment with $\mathrm{NaClO}_{2}$ in $t-\mathrm{BuOH} / \mathrm{H}_{2} \mathrm{O}^{60}$ gave the carboxylic acid which upon immediate treatment with $\mathrm{HCl}$ in $\mathrm{MeOH}$ (to remove the acetonide 210 and induce lactone formation) gave (+)-clivonine (1), with spectroscopic data identical to that reported for the natural product, in $21 \%$ yield. The overall yield from 7-arylindoline (+)-6 was therefore $3.8 \%$ over the 8 steps [i.e. $1.9 \%$ over the 15 steps from enone (+)-5)] although there is clearly room for 215 optimisation of the final oxidation steps.

We also explored routes towards clivonine (1) from arylindoline ( \pm )-6 that did not involve the introduction of $\mathrm{C7}$ (ultimately the lactone carbonyl carbon) concomitant with closure to a lycorine-type galanthan ring system [ $c f$. the aforementioned 220 Pictet-Spengler $(\mathbf{6} \rightarrow \mathbf{2 0})$ and Bischler-Napieralski reactions (18 $\rightarrow$ 19)] but rather by initial $N$-functionalisation and then formylation or sequential bromination/carbonylation. Our inital plan was to start by introducing the methyl group onto the nitrogen then perform $S_{E} A r$ functionalisaton of the aryl ring-A 225 but when this proved problematic, due to the nucleophilicity of the tertiary amine function, we opted to protect the nitrogen as an amide during the $\mathrm{S}_{E} \mathrm{Ar}$ functionalisaton. This allowed bromination and subsequent carbonylation and provided efficient 
access to an $N$-benzyl analogue of clivonine $\mathbf{3 1}$ but left a difficult 230 final FGI to replace this group with the $N$-methyl group found in $( \pm)$-clivonine itself (Scheme 5).
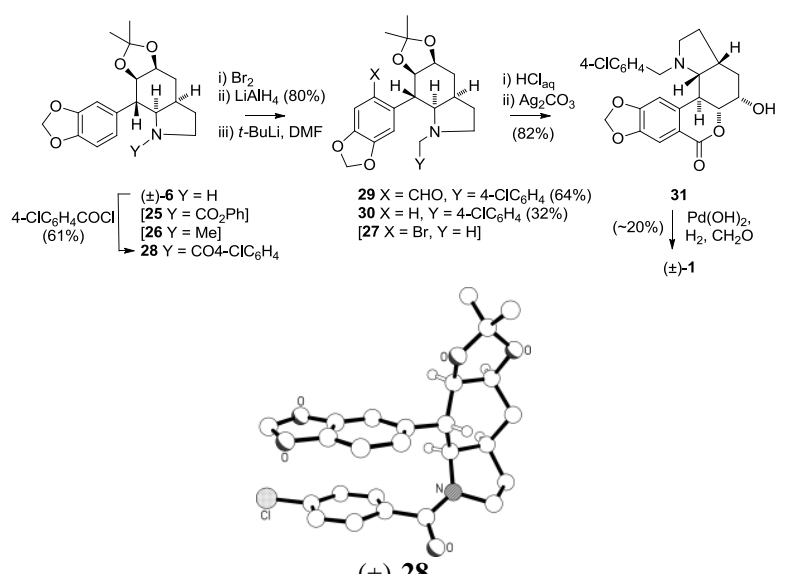

$( \pm)-28$

Scheme 5. Synthesis of clivonine ( \pm -1 directly from 7-arylhydrindane $( \pm)-6$ and the molecular structure of 4-chlorobenzamide ( \pm )-28 (X-ray).

Protection of 7-arylindoline ( \pm )-6 with phenylchloroformate gave the corresponding carbamate $\mathbf{2 5}$ in $72 \%$ yield. This compound could be reduced cleanly to the $N$-methyl derivative 23526 using $\mathrm{LiAlH}_{4}$ (99\% yield). However, all attempts to effect electrophilic formylation (e.g. using $\mathrm{DMF} / \mathrm{POCl}_{3}$ or $\mathrm{TiCl}_{4} / \mathrm{Cl}_{2} \mathrm{CHOMe}$ ) or bromination of this compound (e.g. using $\mathrm{Br}_{2}$ or NBS) gave intractable mixtures of multiple products apparently arising from reaction of these reagents at nitrogen. To 240 circumvent this, we opted to perform bromination on the carbamate prior to reduction to the $N$-methyl derivative. Our initial attempts at bromination utilised methyl carbamate $\mathbf{1 8}$ and also resulted in very unpromising mixtures of multiple products. By contrast, the bromination of phenyl carbamate 25 using $\mathrm{Br}_{2}$ 245 was somewhat cleaner but the desired brominated $N$-methyl derivative 27 was obtained in just $21 \%$ yield following $\mathrm{LiAlH}_{4}$ reduction due to partial $\mathrm{C}-\mathrm{Br}$ bond hydrogenolysis concomitant with the carbamate reduction. Intrigued by the difference in reactivity towards electrophilic bromination between the alkyl 250 and aryl carbamate derivatives $\mathbf{1 8}$ and $\mathbf{2 5}$, and cognisant that the latter (and also the structurally related phenyl carbamate derivatives 21 and 22, Scheme 4) showed distinct peak-broadning in their ${ }^{1} \mathrm{H}$ NMR spectra at RT which we attributed to restricted mutual rotation of the carbamate and 7-indoline aryl groups, ${ }^{* * *}$

255 we hypothesised that intramolecular $\pi-\pi$ interactions could be positively influencing the outcome of our attempted bromination reactions. To test this notion, $N$-(4-chlorobenzoyl) derivative $\mathbf{2 8}$ was prepared from 7 -arylindoline $\mathbf{6}$ in $61 \%$ yield. A single crystal $\mathrm{X}$-ray structure determination on this compound revealed that in 260 the solid state the amide and 7-indoline aryl groups are orientated for face-to-face $\pi-\pi$ stacking (Scheme 5). Pleasingly, amide 28 underwent relatively clean bromination to give the corresponding bromo- $N$-(4-chlorobenzyl) derivative in $80 \%$ yield following amide reduction with $\mathrm{LiAlH}_{4}$. Subsequent bromine-lithium 265 exchange using $t$ - BuLi at $-78{ }^{\circ} \mathrm{C}$ followed by reaction with $\mathrm{DMF}$ afforded a separable mixture of aldehyde 29 (64\% yield) and the debrominated product $30 \quad(32 \%$ yield $)$. Upon acetonide deprotection using aqueous $\mathrm{HCl}$, a crude lactol/aldehyde containing mixture was obtained which on treatment with 270 Fetizon's reagent (i.e. $\mathrm{Ag}_{2} \mathrm{CO}_{3}$-Celite $\left.{ }^{\circledR}\right)^{61}$ furnished lactone 31 in $82 \%$ yield. Finally, $\mathrm{N}$-debenzylation/methylation of lactone $\mathbf{3 1}$ was accomplished using Overman's one-pot procedure ${ }^{62}$ to afford ( \pm )-clivonine (1) in an unoptimised $\sim 20 \%$ yield (by ${ }^{1} \mathrm{H}$ NMR). The overall yield from 7-arylindoline ( \pm )-6 via this route 275 was therefore $\sim 5.1 \%$ over the 6 steps [i.e. $\sim 2.5 \%$ over the 13 steps from enone $( \pm)-5)]$ although again there is clearly room for optimisation of the final $\mathrm{N}$-debenzylation/methylation procedure.

\section{Conclusions}

In summary, we have reported the total synthesis of the 280 lycorine-type Amaryllidaceae alkaloid clivonine (1) via two routes. Both routes employ previously reported 7-arylhydrindane 6, which is prepared using a retro-Cope elimination as the key step, as an intermediate. They differ in the method employed for subsequent introduction of what becomes the ring-B lactone 285 carbonyl carbon (C7). The first route described (Scheme 4) features a Bischler-Napieralski reaction for this transformation and constitutes the first asymmetric synthesis of natural clivonine $[(+)-\mathbf{1}]$ as it was prepared from enantiomerically pure enone (+)-5 (15 steps, $1.9 \%$ overall yield). The second route described 290 (Scheme 5), which employs bromination/carbonylation for C7 introduction, however provides more efficient access to racemic clivonine (1) from enone ( \pm )-5 on both a step-count and yield basis (13 steps, $\sim 2.5 \%$ overall yield). Moreover, this second approach potentially provides access to a range of clivonine 295 analogues having alternative $\mathrm{N}$-substituents following $\mathrm{N}$ debenzylation. The fact that neither synthesis exceeds the efficiency of our previously described route to $( \pm)$-clivonine (1) utilising a biomimetic ring-switch approach (Scheme 1; 12 steps, $6.1 \%$ overall yield) underscores the efficiency of that approach 300 but also reflects to some extent the fact that the final steps of these latest approaches remain to be fully optimised.

\section{Experimental Section}

General Directions. All reactions were performed under anhydrous conditions and an inert atmosphere of $\mathrm{N}_{2}$ in the oven or flame dried glassware. Yields refer to 305 chromatographically and spectroscopically $\left({ }^{1} \mathrm{H}\right.$ NMR) homogenous materials, unless otherwise indicated. Reagents were used as obtained from commercial sources or purified according to known procedures. ${ }^{63}$ Flash chromatography (FC) was carried out using Merck Kiesegel $60 \mathrm{~F}_{254}$ (230-400 mesh) silica gel. Only distilled solvents were used as eluents. Thin layer chromatography (TLC) was performed on Merck 310 DC-Alufolien or glass plates pre-coated with silica gel $60 \mathrm{~F}_{254}$ which were visualised either by quenching of ultraviolet fluorescence $\left(\lambda_{\max }=254 \mathrm{~nm}\right)$ or by charring with $5 \% \mathrm{w} / \mathrm{v}$ phosphomolybdic acid in $95 \% \mathrm{EtOH}, 10 \% \mathrm{w} / \mathrm{v}$ ammonium molybdate in $1 \mathrm{M} \mathrm{H}_{2} \mathrm{SO}_{4}$, or $10 \% \mathrm{KMnO}_{4}$ in $1 \mathrm{M} \mathrm{H}_{2} \mathrm{SO}_{4}$. Observed retention factors $\left(\mathrm{R}_{f}\right)$ are quoted to the nearest 0.05 . All reaction solvents were distilled before use and stored over 315 activated $4 \AA$ molecular sieves, unless otherwise indicated. Anhydrous $\mathrm{CH}_{2} \mathrm{Cl}_{2}$ was obtained by refluxing over $\mathrm{CaH}_{2}$. Anhydrous THF and $\mathrm{Et}_{2} \mathrm{O}$ were obtained by distillation, immediately before use, from sodium/benzophenone ketyl under an inert atmosphere of $\mathrm{N}_{2}$. Anhydrous DMF was obtained by distillation from $\mathrm{CaH}_{2}$ under reduced pressure. Ethylene glycol was distilled immediately prior to use. Petrol 320 refers to the fraction of light petroleum boiling between $40-60{ }^{\circ} \mathrm{C}$. NMR $J$ values are given in Hz. High Resolution Mass Spectrometry (HRMS) measurements are valid to $\pm 5 \mathrm{ppm}$. Microanalyses were performed by Mr Stephen Boyer (Microanalysis Service, London Metropolitan University).

Detailed procedures for the syntheses of $( \pm)-\mathbf{5},( \pm)-6,( \pm)-7,( \pm)-\mathbf{1 0},( \pm)-\mathbf{1 2},( \pm)-\mathbf{1 4}$, $325( \pm)-\mathbf{1 5}$ and $( \pm)-\mathbf{1 7}$ can be found in the Supplementary Material for ref. $^{23}$ Enantiomerically pure $(+)-(5 S, 6 S)-5^{35}$ \{white needles: Mp $82-83{ }^{\circ} \mathrm{C}$ 
(EtOAc/pentane), $[\alpha]_{\mathrm{D}}+107.0\left(\right.$ c. $\left.\left.1.00, \mathrm{MeOH}, 20{ }^{\circ} \mathrm{C}\right)\right\}$ was prepared from enantiopure chlorodiene derivative (+)-(2S,3S)-1-chloro-4,6-cyclohexadiene-2,3-diol (CSS-Almac, Craigavon, Northern Ireland) via a modification ${ }^{34}$ of the method 330 described by Oppolzer. ${ }^{35}$ The subsequent enantiopure intermediates 7, 10, 12, 14 15, 17 and $\mathbf{6}$ were prepared in an identical fashion to the above racemates, in the same yields and had identical spectroscopic characteristics but the following physical properties and optical rotations: $(+)-(1 S, 5 S, 6 S)-7^{35}$ white solid: Mp 115-116 ${ }^{\circ} \mathrm{C}\left(\mathrm{Et}_{2} \mathrm{O}\right),[\alpha]_{\mathrm{D}}+6.5\left(c .1 .00, \mathrm{MeOH}, 20{ }^{\circ} \mathrm{C}\right) ;(+)-(3 R, 5 S, 6 R)-10$ colourless oil: $[\alpha]_{\mathrm{D}}$ $335+19.5$ (c. $\left.1.00, \mathrm{CHCl}_{3}, 20{ }^{\circ} \mathrm{C}\right) ;(+)-(3 R, 5 S, 6 R)-\mathbf{1 2}$ white solid: $\mathrm{Mp} 79{ }^{\circ} \mathrm{C}$ $\left(\mathrm{Et}_{2} \mathrm{O} /\right.$ pentane $),[\alpha]_{\mathrm{D}}+26.4\left(\right.$ c. $\left.0.95, \mathrm{CHCl}_{3}, 20{ }^{\circ} \mathrm{C}\right) ;(+)-(E, 3 R, 5 S, 6 R)-14$ white solid: $\mathrm{Mp} 139{ }^{\circ} \mathrm{C}\left(\mathrm{Et}_{2} \mathrm{O} /\right.$ pentane $),[\alpha]_{\mathrm{D}}+11.4\left(\right.$ c. $\left.0.98, \mathrm{CHCl}_{3}, 20{ }^{\circ} \mathrm{C}\right) ;(-)-(Z, 3 R, 5 S, 6 R)-\mathbf{1 4}$ white solid: $\mathrm{Mp} 141{ }^{\circ} \mathrm{C}\left(\mathrm{Et}_{2} \mathrm{O} /\right.$ pentane), $[\alpha]_{\mathrm{D}}-9.8$ (c. $\left.0.84, \mathrm{CHCl}_{3}, 20{ }^{\circ} \mathrm{C}\right) ;(+)$ (3R,5S,6R)-15 white solid: $\mathrm{Mp} 99-102{ }^{\circ} \mathrm{C}\left(\mathrm{MeOH}_{\mathrm{Et}} \mathrm{O}\right),[\alpha]_{\mathrm{D}}+14.6$ (c. 1.16, $\left.340 \mathrm{CHCl}_{3}, 20{ }^{\circ} \mathrm{C}\right) ;(+)-(3 \mathrm{a} R, 5 S, 6 R, 7 S, 7 \mathrm{a} R)-17$ white solid: $\mathrm{Mp} 61-65{ }^{\circ} \mathrm{C}\left(\mathrm{Et}_{2} \mathrm{O}\right),[\alpha]_{\mathrm{D}}$ $+65.0\left(\right.$ c. $\left.0.50, \mathrm{CHCl}_{3}, 20^{\circ} \mathrm{C}\right) ;(+)-(3 \mathrm{a} R, 5 S, 6 R, 7 S, 7 \mathrm{a} R)-6$ colourless oil, $[\alpha]_{\mathrm{D}}+13.3$ (c. $0.25, \mathrm{CHCl}_{3}, 20^{\circ} \mathrm{C}$ ).

\section{Clivonine (1).}

Method $1[(+)-1$ via oxidation then hydrolysis of aldehyde (+)-24]: To a solution of 345 aldehyde (+)-24 $(11 \mathrm{mg}, 0.031 \mathrm{mmol})$ in $t$ - $\mathrm{BuOH}(1.3 \mathrm{~mL})$ was added 2-methyl-2butene $(167 \mu \mathrm{L}, 111 \mathrm{mg}, 1.578 \mathrm{mmol})$ dropwise followed by a solution of $85 \% \mathrm{w} / \mathrm{w}$ $\mathrm{NaClO}_{2}(33 \mathrm{mg}, 0.31 \mathrm{mmol})$ and $\mathrm{NaH}_{2} \mathrm{PO}_{4} \cdot 2 \mathrm{H}_{2} \mathrm{O}(27 \mathrm{mg}, 0.173 \mathrm{mmol})$ in $\mathrm{H}_{2} \mathrm{O}(500$ $\mu \mathrm{L})$. The reaction mixture was stirred for $14 \mathrm{~h}$ at $\mathrm{RT}$, partitioned between $\mathrm{CH}_{2} \mathrm{Cl}_{2}$ $(10 \mathrm{~mL})$ and brine $(10 \mathrm{~mL})$, the phases separated, the aqueous phase reextracted with

$350 \mathrm{CH}_{2} \mathrm{Cl}_{2}(4 \times 10 \mathrm{~mL})$, the combined organic phases dried over $\mathrm{Na}_{2} \mathrm{SO}_{4}$ and concentrated in vacuo. The solid yellow residue (crude acid, $\sim 10 \mathrm{mg}$ ) was then dissolved in $\mathrm{MeOH}(2 \mathrm{~mL})$ and cooled to $0{ }^{\circ} \mathrm{C}$. To this solution was added dropwise a solution of acetyl chloride $(200 \mu \mathrm{L})$ in $\mathrm{MeOH}(2 \mathrm{~mL})$ also at $0{ }^{\circ} \mathrm{C}$. The resulting solution was stirred for $10 \mathrm{~min}$ at $0{ }^{\circ} \mathrm{C}$ and then $4 \mathrm{~h}$ at $\mathrm{RT}$ before concentrating in

355 vacuo. The residue was dissolved in $\mathrm{CH}_{2} \mathrm{Cl}_{2}(10 \mathrm{~mL})$, washed with sat. aq. $\mathrm{NaHCO}_{3}$, $(15 \mathrm{~mL})$, the phases separated, the aqueous phase reextracted with $\mathrm{CH}_{2} \mathrm{Cl}_{2}(5 \times 5$ $\mathrm{mL}$ ) and the combined organic phases dried over $\mathrm{Na}_{2} \mathrm{SO}_{4}$ and concentrated in vacuo. The residue was purified by $\mathrm{FC}\left(\mathrm{SiO}_{2}\right.$; pentane/ $\mathrm{Et}_{2} \mathrm{O} / \mathrm{MeOH}\left(\mathrm{NH}_{3}\right.$ sat. $\left.), 50: 45: 5\right]$ to give clivonine $[(+)-\mathbf{1}]^{13}, 15,18,19,64,65$ as a colourless solid $(2 \mathrm{mg}, 21 \%) .{ }^{23} \mathrm{Mp} \mathrm{198-200}$

$360{ }^{\circ} \mathrm{C}$ (Lit. $\left.199-200{ }^{\circ} \mathrm{C}\right)^{13} ; \mathrm{R}_{f} 0.7\left(\mathrm{CHCl}_{3} / \mathrm{MeOH}, 9: 1\right) ; v_{\max } / \mathrm{cm}^{-1}$ (neat): 1036, 1274 , $1477,1710,2924,3420 ; \delta_{\mathrm{H}}\left(400 \mathrm{MHz}, \mathrm{CDCl}_{3}\right): 1.80(1 \mathrm{H}, \mathrm{ddd}, J 15.3,6.3,3.9$, $\left.\mathrm{C}_{4} H \mathrm{H}\right), 2.05-2.15\left(1 \mathrm{H}, \mathrm{m}, \mathrm{C}_{4} \mathrm{H} H\right), 2.22-2.31\left(2 \mathrm{H}, \mathrm{m}, \mathrm{C}_{2} H \mathrm{H} \& \mathrm{C}_{3} H \mathrm{H}\right), 2.48-2.58$ $\left(5 \mathrm{H}, \mathrm{m}, \mathrm{NCH}_{3}, \mathrm{C}_{3 \mathrm{a}} \mathrm{H}, \mathrm{C}_{3} \mathrm{HH}\right), 2.89\left(1 \mathrm{H}, \mathrm{dd}, J 10.0,6.7, \mathrm{C}_{11 \mathrm{c}} \mathrm{H}\right), 3.22(1 \mathrm{H}, \mathrm{dd}, J 12.3$, $\left.10.0, \mathrm{C}_{11 \mathrm{~b}} \mathrm{H}\right), 3.25-3.32\left(1 \mathrm{H}, \mathrm{m}, \mathrm{C}_{2} H \mathrm{H}\right), 4.09\left(1 \mathrm{H}, \mathrm{dd}, J 12.3,2.7, \mathrm{C}_{5 \mathrm{a}} \mathrm{H}\right), 4.24(1 \mathrm{H}$,

$\left.365 \mathrm{dd}, J 6.3,2.7, \mathrm{C}_{5} \mathrm{H}\right), 6.02\left(2 \mathrm{H}, A B, J 5.0, \mathrm{OCH}_{2} \mathrm{O}\right), 7.46\left(1 \mathrm{H}, \mathrm{s}, \mathrm{C}_{8} \mathrm{H}\right), 7.74(1 \mathrm{H}, \mathrm{s}$, $\left.\mathrm{C}_{11} \mathrm{H}\right), \mathrm{OH}$ absent; ); $\delta_{\mathrm{C}}\left(125 \mathrm{MHz}, \mathrm{CDCl}_{3}\right) 28.73$ (t), 30.82 (t), 33.12 (d), 33.43 (d), $45.22(\mathrm{q}), 52.95(\mathrm{t}), 67.41$ (d), 69.50 (d), 81.81 (d), $101.82(\mathrm{t}), 107.15(\mathrm{~d}), 109.34$ (d), 118.69 (s), 140.77 (s), 146.68 (s), 152.67 (s), 164.68 (s); m/z (ESI $\left.{ }^{+}\right) 318\left(\mathrm{MH}^{+}\right.$, $28 \%$ ), 282 (70); Found $\mathrm{m} / z \mathrm{MH}^{+}, 318.1345, \mathrm{C}_{17} \mathrm{H}_{20} \mathrm{NO}_{5}$, requires $318.1341(\Delta=1.3$

$370 \mathrm{ppm})$. Unfortunately, we were unable to obtain a optical rotation on our synthetic sample; the natural material is reported to have $[\alpha]_{\mathrm{D}}+41.2\left(\text { c. } 1.11, \mathrm{CHCl}_{3}, 23{ }^{\circ} \mathrm{C}\right)^{13}$.

Method $2[( \pm)-1$ via hydrogenolysis/reductive amination of 4-chlorobenzylamine ( \pm )-31]: Under an atmosphere of $\mathrm{H}_{2}$, Pearlman's catalyst $\left[\mathrm{Pd}(\mathrm{OH})_{2} / \mathrm{C}\right](5 \mathrm{mg}, 10$ $\mathrm{mol} \%)$ and formaldehyde $(37 \%$ aq., $2 \mathrm{~mL}$ ) were added to a solution of 4 -

375 chlorobenzylamine ( \pm -31 (10 mg, $0.02 \mathrm{mmol})$ in EtOAc/MeOH (1:10, $2 \mathrm{~mL})$ at RT. After stirring for $5 \mathrm{~h}$, the suspension was filtered and concentrated in vacuo to give a mixture of compounds including clivonine $\left[( \pm)-\mathbf{1}, \sim 20 \%\right.$ by ${ }^{1} \mathrm{H}$ NMR $]$ which was not isolated.

$\left(5 S^{*}, 6 R^{*}\right)-5,6-\mathrm{Di}-O$-isopropyldiene-1-[3,4-(methylenedioxy)phenyl]cyclohex-1,3380 diene 8. In a flame-dried 2-neck flask equipped with a condenser, $n$-BuLi $(0.19 \mathrm{~mL}$, $2.26 \mathrm{M}$ in hexanes, $0.43 \mathrm{mmol}$ ) was added dropwise to a solution of acetate $( \pm)-7$ (120 mg, $0.36 \mathrm{mmol})$ in THF $(12 \mathrm{~mL})$ at $-78^{\circ} \mathrm{C}$. After stirring for $10 \mathrm{~min}$, freshly distilled TMSCl $(55 \mu \mathrm{l}, 0.433 \mathrm{mmol})$ was added dropwise. After stirring for further $10 \mathrm{~min}$, the dry ice bath was removed and the solution was allowed to reach RT over $3853 \mathrm{~h}$. The solution was then refluxed for $7 \mathrm{~h}$, cooled to RT and partitioned between $\mathrm{Et}_{2} \mathrm{O}(50 \mathrm{~mL})$ and brine $(50 \mathrm{~mL})$. The organic phase was extracted with $\mathrm{Et}_{2} \mathrm{O}(3 \times 20$ $\mathrm{mL}$ ). The combined organic phases were dried over $\mathrm{MgSO}_{4}$, filtered and concentrated in vacuo to give a dark orange solid, which was purified by $\mathrm{FC}\left(\mathrm{SiO}_{2}\right.$; $\mathrm{Et}_{2} \mathrm{O} /$ petrol, $\left.4: 1\right)$ to give diene ( \pm )-8 as a light yellow solid (93 mg, 95\%): Mp 65.7-

$39069.2{ }^{\circ} \mathrm{C}\left(\mathrm{Et}_{2} \mathrm{O} /\right.$ petrol $) ; \mathrm{R}_{f} 0.65\left(\mathrm{Et}_{2} \mathrm{O} /\right.$ petrol, $\left.1: 1\right) ; v_{\max } / \mathrm{cm}^{-1}(\mathrm{KBr}) 1039,1248,1490$, $1506,1608,1732,2895,2984 ; \delta_{\mathrm{H}}\left(\mathrm{CDCl}_{3}, 400 \mathrm{MHz}\right) 1.43\left(3 \mathrm{H}, \mathrm{s}, \mathrm{CH}_{3}\right), 1.51(3 \mathrm{H}, \mathrm{s}$, $\left.\mathrm{CH}_{3}\right), 4.93\left(2 \mathrm{H}\right.$, br s, $\left.\mathrm{C}_{5} \mathrm{H} \& \mathrm{C}_{6} \mathrm{H}\right), 5.89\left(1 \mathrm{H}, \mathrm{dd}, J 9.0,1.0, \mathrm{C}_{4} \mathrm{H}\right), 6.00(2 \mathrm{H}, \mathrm{s}$, $\left.\mathrm{OCH}_{2} \mathrm{O}\right), 6.12\left(1 \mathrm{H}, \mathrm{dd}, J 9.0,6.0, \mathrm{C}_{3} \mathrm{H}\right), 6.35\left(1 \mathrm{H}, \mathrm{d}, J 6.0, \mathrm{C}_{2} \mathrm{H}\right), 6.85(1 \mathrm{H}, \mathrm{d}, J 7.5$ $\left.\mathrm{C}_{\mathrm{ar}} \mathrm{H}\right), 7.13\left(2 \mathrm{H}, \mathrm{m}, 2 \times \mathrm{C}_{\mathrm{ar}} \mathrm{H}\right) ; \delta_{\mathrm{C}}\left(\mathrm{CDCl}_{3}, 100 \mathrm{MHz}\right) 25.34(\mathrm{q}), 26.97$ (q), 72.19 (d), 39573.05 (d), 101.13 (t), 105.78 (d), 106.10 (d), 108.38 (d), 119.62 (d), 119.82 (d) 123.47 (d), 125.79 (d), 133.14 (s), 134.50 (s), 147.35 (s), 147.98 (s); $\mathrm{m} / z\left(\mathrm{CI}^{+}\right) 273$ $\left(\mathrm{MH}^{+}, 60\right), 290\left(\mathrm{MNH}_{4}^{+}, 21\right), 215$ (100); Found: $\mathrm{m} / z \mathrm{MH}^{+}, 273.1118, \mathrm{C}_{16} \mathrm{H}_{17} \mathrm{O}_{4}$ requires $273.1115(\Delta=+0.5 \mathrm{ppm})$; Calculated for $\mathrm{C}_{16} \mathrm{H}_{16} \mathrm{O}_{4} \mathrm{C}, 70.57 \%$; $\mathrm{H}, 5.92 \%$; $\mathrm{O}, 23.50 \%$, found $\mathrm{C}, 70.09 \% ; \mathrm{H}, 5.92 \%$;, $23.99 \%$.

\section{$400\left(3 R^{*}, 5 S^{*}, 6 R^{*}\right)-5,6-\mathrm{Di}-O$-isopropyldiene-3-acetoxy-1-[3,4-}

(methylenedioxy)phenyl]cyclohex-1-ene 9. In a flame-dried 2-neck flask equipped with a condenser, acetate $( \pm)-7(0.30 \mathrm{mg}, 0.88 \mathrm{mmol})$ was dissolved in THF $(10 \mathrm{~mL})$ and cooled to $-78{ }^{\circ} \mathrm{C}$. A solution of LDA $[9 \mathrm{~mL}, 0.10 \mathrm{M}$ in THF, prepared by dropwise addition of $n$ - BuLi (2.5 M in hexanes, 1 equiv) to a stirred solution of 405 diisopropylamine ( 1 equiv) in THF at $0{ }^{\circ} \mathrm{C}$ followed by stirring for $20 \mathrm{~min}$ at $0{ }^{\circ} \mathrm{C}$ ] was then added via syringe and the mixture was stirred at $-78{ }^{\circ} \mathrm{C}$ for $10 \mathrm{~min}$. Freshly distilled TMSCl $(136 \mu \mathrm{l}, 1.06 \mathrm{mmol})$ was added dropwise. After stirring for further $10 \mathrm{~min}$, the dry ice bath was removed and the solution was allowed to reach RT over $3 \mathrm{~h}$. The solution was then refluxed for $12 \mathrm{~h}$, cooled to RT and partitioned with $\mathrm{Et}_{2} \mathrm{O}$ $410(50 \mathrm{~mL})$ and brine $(50 \mathrm{~mL})$. The organic phase was extracted with $\mathrm{Et}_{2} \mathrm{O}(3 \times 20 \mathrm{~mL})$. The combined organic phases were dried over $\mathrm{MgSO}_{4}$, filtered and concentrated in vacuo. The residue was purified by $\mathrm{FC}\left(\mathrm{SiO}_{2} ; \mathrm{Et}_{2} \mathrm{O}\right.$ /petrol, 1:4) to afford rearranged acetate ( \pm )-9 as a white solid (130 mg, 43\%): $\mathrm{Mp} \mathrm{78.5-79.7}{ }^{\circ} \mathrm{C}\left(\mathrm{Et}_{2} \mathrm{O} /\right.$ petrol $) ; \mathrm{R}_{f} 0.7$ $\left(\mathrm{Et}_{2} \mathrm{O} /\right.$ petrol, 1:1); $v_{\max } / \mathrm{cm}^{-1}(\mathrm{KBr}) 1023,1241,1375,1508,1720(\mathrm{C}=\mathrm{O}), 2883,2995$;

$415 \delta_{\mathrm{H}}\left(\mathrm{CDCl}_{3}, 400 \mathrm{MHz}\right) 1.37\left(3 \mathrm{H}, \mathrm{s}, \mathrm{CH}_{3}\right), 1.51\left(3 \mathrm{H}, \mathrm{s}, \mathrm{CH}_{3}\right), 1.80(1 \mathrm{H}, \mathrm{dt}, J$ 12.0, 9.5, $\left.\mathrm{C}_{4} H \mathrm{H}\right), 2.02\left(3 \mathrm{H}, \mathrm{s}, \mathrm{CH}_{3} \mathrm{O}\right), 2.16\left(1 \mathrm{H}, \mathrm{dt}, J 12.0,5.5, \mathrm{C}_{4} \mathrm{HH}\right), 4.33(1 \mathrm{H}, \mathrm{dt}, J 9.5$, $\left.5.5, \mathrm{C}_{5} \mathrm{H}\right), 4.47\left(1 \mathrm{H}, \mathrm{d}, J 5.5, \mathrm{C}_{6} \mathrm{H}\right), 5.34\left(1 \mathrm{H}, \mathrm{m}, \mathrm{C}_{3} \mathrm{H}\right), 5.89\left(2 \mathrm{H}, \mathrm{s}, \mathrm{OCH}_{2} \mathrm{O}\right), 6.06$ $\left(1 \mathrm{H}, \mathrm{d}, J 6.5, \mathrm{C}_{2} \mathrm{H}\right), 6.71\left(1 \mathrm{H}, \mathrm{d}, J 8.5, \mathrm{C}_{\mathrm{ar}} \mathrm{H}\right), 7.01\left(2 \mathrm{H}, \mathrm{m}, 2 \times \mathrm{C}_{\mathrm{ar}} \mathrm{H}\right) ; \delta_{\mathrm{C}}\left(\mathrm{CDCl}_{3}\right.$, $100 \mathrm{MHz}) 21.25$ (q), 26.39 (q), 28.44 (d), 31.68 (t), 68.36 (d), 72.21 (d), 72.51 (d), 420100.96 (t), 106.74 (d), 108.30 (d), 109.9 (s), 120.15 (d), 126.88 (d), 132.36 (s), 136.09 (s), 147.63 (s), 147.94 (s), 179.69 (s); m/z $\left(\mathrm{CI}^{+}\right) 273\left\{[\mathrm{M}-(\mathrm{AcOH})+\mathrm{H}]^{+}, 56\right\}$; Found: $m / z[\mathrm{M}-(\mathrm{AcOH})+\mathrm{H}]^{+}, 273.1127, \mathrm{C}_{16} \mathrm{H}_{17} \mathrm{O}_{4}$, requires $273.1124(\Delta=+0.3$ ppm); Calculated for $\mathrm{C}_{18} \mathrm{H}_{20} \mathrm{O}_{6}, \mathrm{C}, 65.05 \% ; \mathrm{H}, 6.07 \% ; \mathrm{O}, 28.88 \%$, found $\mathrm{C}$, $64.97 \%$; H, 6.09\%; O, 28.94\%.

\section{$425(3 R *, 5 S *, 6 \mathrm{R} *)-5,6-\mathrm{Di}-\boldsymbol{O}$-isopropylidene-3-(2-oxoethyl)-1-[3,4-}

(methylenedioxy)phenyl]cyclohex-1-ene $12{ }^{23}$ To a stirred solution of methyl ester $( \pm)-10(6.73 \mathrm{~g}, 19.43 \mathrm{mmol})$ in THF $(150 \mathrm{~mL})$ at $0{ }^{\circ} \mathrm{C}$ was added $\mathrm{LiBH}_{4}(68.02 \mathrm{~mL}$, $2 \mathrm{M}$ in THF, $136.04 \mathrm{mmol}$ ) and the mixture warmed to RT. After stirring for $40 \mathrm{~h}$ the reaction was quenched with $\mathrm{H}_{2} \mathrm{O}(100 \mathrm{~mL})$ and the organic phase extracted with 430 EtOAc $(4 \times 100 \mathrm{ml})$. The combined organic phases were washed with brine $(200$ $\mathrm{mL}$ ), dried over $\mathrm{Na}_{2} \mathrm{SO}_{4}$ and concentrated in vacuo to give alcohol ( \pm )-11 as a yellow foam $(5.34 \mathrm{~g}, 86 \%): \mathrm{R}_{f} 0.39\left(\mathrm{Et}_{2} \mathrm{O} /\right.$ petrol, 3:1); $v_{\max } / \mathrm{cm}^{-1}$ (neat) 3427,2924 , $1720,1504,1488,1371,1244,1215,1038,935,749 ; \delta_{\mathrm{H}}\left(\mathrm{CDCl}_{3}, 400 \mathrm{MHz}\right) 1.44$ $\left(1 \mathrm{H}, \mathrm{m}, \mathrm{C}_{4} H \mathrm{H}\right), 1.47\left(3 \mathrm{H}, \mathrm{s}, \mathrm{CH}_{3}\right), 1.52\left(3 \mathrm{H}, \mathrm{s}, \mathrm{CH}_{3}\right), 1.61(1 \mathrm{H}, \mathrm{br} \mathrm{s}, \mathrm{OH}), 1.73(1 \mathrm{H}$, $\left.435 \mathrm{td}, J 13.9,6.5, \mathrm{C}_{3} H \mathrm{H}\right), 1.86\left(1 \mathrm{H}, \mathrm{td}, J 13.5,6.5, \mathrm{C}_{3} \mathrm{H} H\right), 2.01(1 \mathrm{H}$, app dt, $J 12.1$, 4.7, $\left.\mathrm{C}_{4} \mathrm{HH}\right), 2.45\left(1 \mathrm{H}, \mathrm{m}, \mathrm{C}_{3 \mathrm{a}} \mathrm{H}\right), 3.84\left(2 \mathrm{H}, \mathrm{m}, \mathrm{C}_{3 \mathrm{~b}} \mathrm{H}_{2}\right), 4.32(1 \mathrm{H}$, app dt, $J$ 10.5, 5.0, $\left.\mathrm{C}_{5} \mathrm{H}\right), 4.83\left(1 \mathrm{H}, \mathrm{d}, J 5.0, \mathrm{C}_{6} \mathrm{H}\right), 5.98\left(2 \mathrm{H}, \mathrm{s}, \mathrm{OCH}_{2} \mathrm{O}\right), 6.17\left(1 \mathrm{H}, \mathrm{d}, J 2.3, \mathrm{C}_{2} \mathrm{H}\right), 6.82$ $\left(1 \mathrm{H}, \mathrm{d}, J 8.3, \mathrm{C}_{\mathrm{Ar}} \mathrm{H}\right), 7.07-7.10\left(2 \mathrm{H}, 2 \times \mathrm{C}_{\mathrm{Ar}} \mathrm{H}\right) ; \delta_{\mathrm{C}}\left(\mathrm{CDCl}_{3}, 100 \mathrm{MHz}\right) 26.26(\mathrm{q})$, $28.64(\mathrm{q}), 31.54$ (d), 32.48 (t), 38.09 (t), 60.03 (t), 72.38 (d), 74.41 (d), 101.65 (t), 440106.38 (d), 108.24 (d), 108.79 (d), 119.50 (d), 132.91 (d), 133.33 (s), 133.36 (s), 147.00 (s), 147.82 (s); m/z (EI $\left.{ }^{+}\right) 318\left(\mathrm{M}^{+}, 32\right), 260$ (46), 215 (52), 91 (100); Found: $m / z \mathrm{MNH}_{4}{ }^{+}, 336.1811, \mathrm{C}_{18} \mathrm{H}_{26} \mathrm{NO}_{5}$, requires $336.1811(\Delta=0.0 \mathrm{ppm})$. To а suspension of Dess-Martin periodianane $(21.4 \mathrm{~g}, 50.4 \mathrm{mmol})$ in $\mathrm{CH}_{2} \mathrm{Cl}_{2}(60 \mathrm{~mL})$ at RT was added a solution of alcohol 11 (5.34 g, $16.8 \mathrm{mmol})$ in $\mathrm{CH}_{2} \mathrm{Cl}_{2}(20 \mathrm{~mL})$ 445 dropwise via cannula. After stirring for $23.5 \mathrm{~h}$ the reaction mixture was filtered through Celite ${ }^{\circledR}$ and the filtrate treated with $\mathrm{NaHCO}_{3}$ (sat. aq., $100 \mathrm{~mL}$ ). The organic phase was extracted with $\mathrm{CH}_{2} \mathrm{Cl}_{2}(2 \times 50 \mathrm{~mL})$. The combined organic phases were washed with $\mathrm{NaHCO}_{3}$ (sat. aq., $50 \mathrm{~mL}$ ), dried over $\mathrm{Na}_{2} \mathrm{SO}_{4}$ and concentrated in vacuo to give aldehyde $( \pm)-12(5.04 \mathrm{~g}, 95 \%) .{ }^{23} \mathrm{Mp} 80.2-81.3{ }^{\circ} \mathrm{C} ; \mathrm{R}_{f} 0.70$ $450\left(\mathrm{Et}_{2} \mathrm{O} /\right.$ petrol, $\left.1: 1\right) ; v_{\max } / \mathrm{cm}^{-1}\left(\mathrm{CHCl}_{3}\right): 754,850,927,1063,1103,1224,1372,1434$, $1485,1507,1723,3020 ; \delta_{\mathrm{H}}\left(\mathrm{CDCl}_{3}, 400 \mathrm{MHz}\right) 1.41\left(3 \mathrm{H}, \mathrm{s}, \mathrm{CH}_{3}\right), 1.45\left(3 \mathrm{H}, \mathrm{s}, \mathrm{CH}_{3}\right)$, 1.48-1.52 (1H, m, $\left.\mathrm{C}_{4} H \mathrm{H}\right), 1.98\left(1 \mathrm{H}\right.$, app dt, $\left.J 12.6,5.3, \mathrm{C}_{4} \mathrm{HH}\right), 2.66-2.68(2 \mathrm{H}, \mathrm{m}$, $\left.\mathrm{C}_{3} \mathrm{H}_{2}\right), 2.80-2.89\left(1 \mathrm{H}, \mathrm{m}, \mathrm{C}_{3 \mathrm{a}} \mathrm{H}\right), 4.33\left(1 \mathrm{H}, \operatorname{app}\right.$ quintet, $\left.J 5.3, \mathrm{C}_{5} \mathrm{H}\right), 4.78(1 \mathrm{H}, \mathrm{d}, J$ $\left.5.3, \mathrm{C}_{6} \mathrm{H}\right), 5.93\left(2 \mathrm{H}, \mathrm{s}, \mathrm{OCH}_{2} \mathrm{O}\right), 6.03\left(1 \mathrm{H}, \mathrm{d}, J 2.8, \mathrm{C}_{2} \mathrm{H}\right), 6.76\left(1 \mathrm{H}, \mathrm{d}, J 8.7, \mathrm{C}_{\mathrm{Ar}} \mathrm{H}\right)$, 455 7.00-7.03 $\left(2 \mathrm{H}, \mathrm{m}, 2 \times \mathrm{C}_{\mathrm{Ar}} \mathrm{H}\right), 9.83(1 \mathrm{H}, \mathrm{s}, \mathrm{CHO}) ; \delta_{\mathrm{C}}\left(\mathrm{CDCl}_{3}, 100 \mathrm{MHz}\right) 26.16(\mathrm{q})$, 28.49 (q), $29.02(\mathrm{~d}), 31.98(\mathrm{t}), 49.34(\mathrm{t}), 72.25(\mathrm{~d}), 73.89$ (d), $101.05(\mathrm{t}), 106.57(\mathrm{~d})$, 108.24 (d), 108.94 (s), 119.71 (d), 130.47 (d), 133.31 (s), 134.92 (s), 147.16 (s), 147.82 (s), 201.02 (d); $\mathrm{m} / \mathrm{z}\left(\mathrm{EI}^{+}\right) 316\left(\mathrm{M}^{+}, 76 \%\right)$; Found $\mathrm{m} / \mathrm{z} \mathrm{M}^{+}, 316.1318$, $\mathrm{C}_{18} \mathrm{H}_{20} \mathrm{O}_{5}$, requires $\left.316.1305 \Delta=+4.1 \mathrm{ppm}\right)$.

\section{$460\left(3 R^{*}, 5 S^{*}, 6 R^{*}\right)-5,6-\mathrm{Di}-\boldsymbol{O}$-isopropylidene-3-(2-dimethyalcetalethyl)-1-[3,4-} (methylenedioxy)phenyl]cyclohex-1-ene 13. To a solution of aldehyde $( \pm)-12$ (377 $\mathrm{mg}, 1.06 \mathrm{mmol})$ in $\mathrm{MeOH}(15 \mathrm{~mL})$ at $\mathrm{RT}$ was added $\mathrm{NH}_{2} \mathrm{OH} \cdot \mathrm{HCl}(111.12 \mathrm{mg}, 1.59$ mmol), $\mathrm{NaOAc}_{(\mathrm{anh} .)}(140.42 \mathrm{mg}, 1.59 \mathrm{mmol})$ and $\mathrm{MgSO}_{4}(190 \mathrm{mg}, 1.59 \mathrm{~mol})$ and the resulting suspension was then refluxed overnight. $\mathrm{MgSO}_{4}$ was filtered and reaction 465 mixture concentrated in vacuo. The solid residue was then partitioned between $\mathrm{CH}_{2} \mathrm{Cl}_{2}(10 \mathrm{~mL})$ and brine $(5 \mathrm{~mL})$. The organic phase was extracted with $\mathrm{CH}_{2} \mathrm{Cl}_{2}(3$ $\times 10 \mathrm{~mL}$ ). The combined organic phases were dried over $\mathrm{Na}_{2} \mathrm{SO}_{4}$ and concentrated in vacuo. The residue was purified by $\mathrm{FC}\left(\mathrm{SiO}_{2}\right.$; petrol/Et $\left.\mathrm{Et}_{2} \mathrm{O}, 1: 1\right)$ to give acetal $( \pm)$ - 
$\mathbf{1 3}$ as colourless needles (95 mg, 39\%): $\mathrm{Mp} 102.3-104.1{ }^{\circ} \mathrm{C}\left(\mathrm{Et}_{2} \mathrm{O} / \mathrm{petrol}\right) ; \mathrm{R}_{f} 0.75$ $470\left(\mathrm{Et}_{2} \mathrm{O} /\right.$ petrol, 1:1); $v_{\max } / \mathrm{cm}^{-1}(\mathrm{KBr}) 980,1039,1253,1494,1510,1612,1742,2901$, $2959 ; \delta_{\mathrm{H}}\left(\mathrm{CDCl}_{3}, 400 \mathrm{MHz}\right) 1.47\left(3 \mathrm{H}, \mathrm{s}, \mathrm{CH}_{3}\right), 1.52\left(3 \mathrm{H}, \mathrm{s}, \mathrm{CH}_{3}\right), 1.75(1 \mathrm{H}, \mathrm{ddd}, J$ 12.0, 10.5, 6.0, $\left.\mathrm{C}_{4} H \mathrm{H}\right), 1.89\left(2 \mathrm{H}, \mathrm{m}, \mathrm{C}_{3} \mathrm{H}_{2}\right), 2.01\left(1 \mathrm{H}, \mathrm{dt}, J 12.0,5.5, \mathrm{C}_{4} \mathrm{HH}\right), 2.39$ $\left(1 \mathrm{H}, \mathrm{m}, \mathrm{C}_{3 \mathrm{a}} \mathrm{H}\right), 3.38\left(6 \mathrm{H}, \mathrm{s}, 2 \times \mathrm{OCH}_{3}\right), 4.31\left(1 \mathrm{H}, \mathrm{dt}, J 10.5,5.5, \mathrm{C}_{5} \mathrm{H}\right), 4.58(1 \mathrm{H}, \mathrm{t}, J$ $\left.6.0, \mathrm{C}_{2} H \mathrm{H}\right), 4.82\left(1 \mathrm{H}, \mathrm{d}, J 5.5, \mathrm{C}_{6} \mathrm{H}\right), 5.98\left(2 \mathrm{H}, \mathrm{s}, \mathrm{OCH}_{2} \mathrm{O}\right), 6.17(1 \mathrm{H}, \mathrm{d}, J 2.5$,

$\left.475 \mathrm{C}_{2} \mathrm{HH}\right), 6.82\left(1 \mathrm{H}, \mathrm{d}, J 8.5, \mathrm{C}_{\mathrm{ar}} \mathrm{H}\right), 7.09\left(2 \mathrm{H}, \mathrm{m}, 2 \times \mathrm{C}_{\mathrm{ar}} \mathrm{H}\right) ; \delta_{\mathrm{C}}\left(\mathrm{CDCl}_{3}, 100 \mathrm{MHz}\right)$ 26.26 (q), 28.65 (q), $31.16(\mathrm{t}), 32.80(\mathrm{~d}), 38.13(\mathrm{t}), 52.78(\mathrm{~d}), 53.06\left(2 \times \mathrm{CH}_{3}\right), 72.28$ (d), 74.29 (d), 101.03 (t), 102.84 (d), 106.46 (d), 108.26 (d), 108.80 (s), 119.54 (d), $132.62(\mathrm{~s}), 133.55$ (s), 147.01 (s), $147.81(\mathrm{~s}) ; \mathrm{m} / 2\left(\mathrm{CI}^{+}\right) 380\left(\mathrm{MNH}_{4}{ }^{+}, 40\right), 273(100)$; Found: $\mathrm{m} / z \mathrm{MNH}_{4}{ }^{+}, 380.2074, \mathrm{C}_{20} \mathrm{H}_{30} \mathrm{NO}_{6}$, requires $380.2073(\Delta=+0.2 \mathrm{ppm})$; 480 Calculated for $\mathrm{C}_{20} \mathrm{H}_{16} \mathrm{O}_{6} \mathrm{C}, 66.28 \% ; \mathrm{H}, 7.23 \% ; \mathrm{O}, 26.49 \%$, found $\mathrm{C}, 66.53 \% ; \mathrm{H}$ $7.28 \%$; O, 26.19\%. For a single crystal X-ray structure determination on this compound see ESI.

Crystal data for 13: $\mathrm{C}_{20} \mathrm{H}_{26} \mathrm{O}_{6}, \mathrm{M}=362.41$, monoclinic, $\mathrm{P} 2{ }_{1} / \mathrm{c}$ (no. 14), a = 14.5941(4), b = 9.4049(2), c = 13.8121(3) $\AA, \beta=91.949(2)^{\circ}, \mathrm{V}=1894.70(8) \AA^{3}, \mathrm{Z}$ $485=4, D_{\mathrm{c}}=1.270 \mathrm{~g} \mathrm{~cm}^{-3}, \mu(\mathrm{Mo}-\mathrm{K} \alpha)=0.093 \mathrm{~mm}^{-1}, \mathrm{~T}=173 \mathrm{~K}$, colourless plates, Oxford Diffraction Xcalibur 3 diffractometer; 6105 independent measured reflections $\left(R_{\text {int }}=0.0455\right), F^{2}$ refinement, $R_{1}(\mathrm{obs})=0.0448, \mathrm{wR}_{2}(\mathrm{all})=0.1191,3310$ independent observed absorption-corrected reflections $\left[\left|F_{o}\right|>4 \sigma\left(\left|F_{o}\right|\right), 2 \theta_{\max }=64^{\circ}\right]$, 235 parameters. CCDC 792816

$490\left(3 R^{*}, 5 S^{*}, 6 R^{*}\right)-5,6-\mathrm{Di}-\boldsymbol{O}$-isopropylidene-3-(2-dimethylnitronylethyl)-1-[3,4(methylenedioxy)phenyl]cyclohex-1-ene 16. A mixture of oxime isomers ( \pm )-14 (636 mg, $1.92 \mathrm{mmol})$ were transferred into a flask containing $\mathrm{NaBH}_{3} \mathrm{CN}(236 \mathrm{mg}$, $3.84 \mathrm{mmol})$ using dry $\mathrm{MeOH}(80 \mathrm{~mL})$ via a cannula. The suspension was heated gently until a clear solution arised and then cooled to $0{ }^{\circ} \mathrm{C} .6$ drops of methyl orange 495 indicator were added and the solution was titrated rapidly dropwise with a mixture of $\left.\mathrm{MeOH}: \mathrm{HCl}_{\text {(conc.) }} 3.0 \mathrm{~mL}, 10: 1\right)$. The cooling bath was removed and the solution was stirred for $5 \mathrm{~min}$ at RT, cooled again to $0{ }^{\circ} \mathrm{C}$ and treated with $\mathrm{NaOH}(8 \mathrm{~mL}, 2$ M). After stirring for $10 \mathrm{~min}$ at $0{ }^{\circ} \mathrm{C}$, the mixture was partitioned between $\mathrm{CH}_{2} \mathrm{Cl}_{2}$ $(15 \mathrm{~mL})$ and $\mathrm{NaHCO}_{3}$ (sat. aq., $15 \mathrm{~mL}$ ) and extracted with $\mathrm{CH}_{2} \mathrm{Cl}_{2}(4 \times 15 \mathrm{~mL})$. The 500 combined organic phases were dried over $\mathrm{Na}_{2} \mathrm{SO}_{4}$ and concentrated in vacuo. The residue was purified by $\mathrm{FC}\left[\mathrm{SiO}_{2} ; \mathrm{Et}_{2} \mathrm{O} /\right.$ petrol, $1: 1 \rightarrow \mathrm{Et}_{2} \mathrm{O} / \mathrm{MeOH}\left(\mathrm{NH}_{3}\right.$ sat.), 97:3] to give nitrone $( \pm)-16$ as colourless needles $(344 \mathrm{mg}, 48 \%)$ : $\mathrm{Mp} 82.6-88.9{ }^{\circ} \mathrm{C}$ $\left(\mathrm{Et}_{2} \mathrm{O} /\right.$ petrol); $\mathrm{R}_{f} 0.35\left[\mathrm{Et}_{2} \mathrm{O} / \mathrm{MeOH}\left(\mathrm{NH}_{3}\right.\right.$ sat.), 9:1]; $v_{\max } / \mathrm{cm}^{-1}$ (neat) 980, 1041, 1253, $1586(\mathrm{C}=\mathrm{N}-\mathrm{OH}), 2930,2984 ; \delta_{\mathrm{H}}\left(\mathrm{CDCl}_{3}, 400 \mathrm{MHz}\right) 1.45\left(3 \mathrm{H}, \mathrm{s}, \mathrm{CH}_{3}\right), 1.47$ $505\left(1 \mathrm{H}, \mathrm{m}, \mathrm{C}_{4} H \mathrm{H}\right), 1.49\left(3 \mathrm{H}, \mathrm{s}, \mathrm{CH}_{3}\right), 1.98\left(1 \mathrm{H}, \mathrm{dt}, J 12.5,5.0, \mathrm{C}_{4} H \mathrm{H}\right), 2.05-2.22(2 \mathrm{H}$, $\left.\mathrm{m}, \mathrm{C}_{3 \mathrm{~b}} \mathrm{H} \mathrm{H} \mathrm{C}_{3 \mathrm{a}} \mathrm{H}\right), 2.14\left(3 \mathrm{H}, \mathrm{s}, \mathrm{CH}_{3}\right), 2.16\left(3 \mathrm{H}, \mathrm{s}, \mathrm{CH}_{3}\right), 2.38\left(1 \mathrm{H}, \mathrm{m}, \mathrm{C}_{3 \mathrm{~b}} \mathrm{HH}\right), 3.98$ $\left(2 \mathrm{H}, \mathrm{m}, \mathrm{CH}_{2}\right), 4.29\left(1 \mathrm{H}, \mathrm{td}, J 10.5,5.0, \mathrm{C}_{5} \mathrm{H}\right), 4.79\left(1 \mathrm{H}, \mathrm{d}, J 5.0, \mathrm{C}_{6} \mathrm{H}\right), 5.96(2 \mathrm{H}, \mathrm{s}$, $\left.\mathrm{OCH}_{2} \mathrm{O}\right), 6.12\left(1 \mathrm{H}, \mathrm{d}, J 2.0, \mathrm{C}_{2} \mathrm{H}\right), 6.79\left(1 \mathrm{H}, \mathrm{dd}, J 8.5,1.0, \mathrm{C}_{\mathrm{ar}} \mathrm{H}\right), 7.05(1 \mathrm{H}, \mathrm{d}, 8.5$, $\left.\mathrm{C}_{\mathrm{ar}} \mathrm{H}\right), 7.07\left(1 \mathrm{H}, \mathrm{d}, 1.0, \mathrm{C}_{\mathrm{ar}} \mathrm{H}\right) ; \delta_{\mathrm{C}}\left(\mathrm{CDCl}_{3}, 100 \mathrm{MHz}\right) 19.96(\mathrm{q}), 20.37(\mathrm{q}), 26.08(\mathrm{q})$, $51028.53(\mathrm{q}), 32.08(\mathrm{t}), 32.51(\mathrm{~d}), 32.63(\mathrm{t}), 56.38(\mathrm{t}), 72.36(\mathrm{~d}), 74.02(\mathrm{~d}), 100.97(\mathrm{t})$ 106.51 (d), 108.18 (d), 108.78 (s), 119.53 (d), 131.25 (d), 133.24 (s), 134.30 (s), 143.38 (s), 147.05 (s), 147.75 (s); $m / z\left(\mathrm{CI}^{+}\right) 374\left(\mathrm{MH}^{+}, 100\right)$; Found: $m / z \mathrm{MH}^{+}$, 374.1969, $\mathrm{C}_{21} \mathrm{H}_{28} \mathrm{NO}_{5}$, requires $374.1967(\Delta=+0.5 \mathrm{ppm})$; Calculated for $\mathrm{C}_{21} \mathrm{H}_{27} \mathrm{NO}_{5}$ $\mathrm{C}, 67.54 \%$; H, 7.29\%; N 3.75\%; O, 21.42\%, found C, 67.58\%; H, 7.31\%; N, 3.70;

$515 \mathrm{O}, 21.41 \% \%$. For a single crystal X-ray structure determination on this compound see ESI.

Crystal data for 16: $\mathrm{C}_{21} \mathrm{H}_{27} \mathrm{NO}_{5} \cdot \mathrm{H}_{2} \mathrm{O}, \mathrm{M}=391.45$, monoclinic, $\mathrm{P} 2_{1} / \mathrm{n}$ (no. 14), $\mathrm{a}=$ 7.2818(1), $\mathrm{b}=33.8728(3), \mathrm{c}=8.3252(1) \AA, \beta=104.148(1)^{\circ}, \mathrm{V}=1991.17(4) \AA^{3}, \mathrm{Z}$ $=4, D_{c}=1.306 \mathrm{~g} \mathrm{~cm}^{-3}, \mu(\mathrm{Cu}-\mathrm{K} \alpha)=0.784 \mathrm{~mm}^{-1}, \mathrm{~T}=173 \mathrm{~K}$, colourless blocks, 520 Oxford Diffraction Xcalibur PX Ultra diffractometer; 3808 independent measured reflections $\left(R_{\text {int }}=0.0259\right), F^{2}$ refinement, $R_{1}(\mathrm{obs})=0.0424, \mathrm{wR}_{2}(\mathrm{all})=0.1248,3426$ independent observed absorption-corrected reflections $\left[\left|\mathrm{F}_{\mathrm{o}}\right|>4 \sigma\left(\left|\mathrm{F}_{\mathrm{o}}\right|\right), 2 \theta_{\max }=143^{\circ}\right]$, 263 parameters. CCDC 792817

\section{(3aR,5S,6R,7S,7aR)-5,6-Di- $O$-isopropylidene-1-carbomethoxy-7-[3,4-}

525 (methylenedioxy)phenyl]-2,3,3a,4,5,6,7,7a-octahydroindole $\mathbf{1 8}$. To a stirred solution of amine (+)-6 $(13 \mathrm{mg}, 0.041 \mathrm{mmol})$ and a crystal of 4-DMAP in $\mathrm{CH}_{2} \mathrm{Cl}_{2}(2$ $\mathrm{mL})$ at $0{ }^{\circ} \mathrm{C}$ were added sequentially and dropwise triethylamine $(17 \mu \mathrm{L}, 12.5 \mathrm{mg}$, $0.12 \mathrm{mmol}$ ) and then a solution of methylchloroformate in $\mathrm{CH}_{2} \mathrm{Cl}_{2}(100 \mu \mathrm{L}, 1 \mathrm{M}, 0.1$ mmol). The solution was stirred for $2 \mathrm{~h}$ at $0{ }^{\circ} \mathrm{C}$ and for $16 \mathrm{~h}$ at RT. The reaction

530 mixture was partitioned between $\mathrm{CH}_{2} \mathrm{Cl}_{2}$ and sat. aq. $\mathrm{NaHCO}_{3}$, the aqueous phase extracted with $\mathrm{CH}_{2} \mathrm{Cl}_{2}(3 \times 10 \mathrm{~mL})$, the combined organic phases washed with sat. aq. $\mathrm{NH}_{4} \mathrm{Cl}(10 \mathrm{~mL})$, the aqueous phase extracted with $\mathrm{CH}_{2} \mathrm{Cl}_{2}(3 \times 20 \mathrm{~mL})$, the combined organic phases dried over $\mathrm{Na}_{2} \mathrm{SO}_{4}$ and the solvent evaporated in vacuo. The residue was purified by $\mathrm{FC}\left(\mathrm{SiO}_{2} ; \mathrm{Et}_{2} \mathrm{O} /\right.$ pentane, 1:1) to give methyl carbamate

$535(+)-18$ as a white solid $(15.0 \mathrm{mg}, 98 \%): \mathrm{Mp} 49{ }^{\circ} \mathrm{C}\left(\mathrm{Et}_{2} \mathrm{O} /\right.$ pentane); $[\alpha]_{\mathrm{D}}+17.7(c$ $\left.0.88, \mathrm{CHCl}_{3}\right) ; v_{\max } / \mathrm{cm}^{-1}\left(\mathrm{CHCl}_{3}\right) 3007,2952,1683,1604,1504,1491,1451,1383$, $1234,1206,1120,1074,1043,938,864,779,745 ; \delta_{\mathrm{H}}\left(\mathrm{CDCl}_{3}, 400 \mathrm{MHz}\right) 1.31(3 \mathrm{H}$, s), $1.45(3 \mathrm{H}, \mathrm{s}), 1.83(2 \mathrm{H}, \mathrm{m}), 2.12(2 \mathrm{H}, \mathrm{m}), 2.38(1 \mathrm{H}$, sextet, $J$ 8), $2.64(1 \mathrm{H}, \mathrm{dd}, J$
10.5, 11.5), 3.21 (3H, broad s), $3.34(1 \mathrm{H}, \mathrm{m}), 3.63(1 \mathrm{H}, \mathrm{m}), 3.97(1 \mathrm{H}, \mathrm{t}, J 8.5), 4.30$ $540(1 \mathrm{H}, \mathrm{m}), 4.40(1 \mathrm{H}, \mathrm{dd}, J 10.5,6.5), 5.91\left(2 \mathrm{H}, \mathrm{AB}_{\mathrm{q}}, J 1.5\right), 6.67(1 \mathrm{H}, \mathrm{dd}, J 6.5,1)$, $6.74\left(1 \mathrm{H}, \mathrm{d}, J\right.$ 8), $6.78(1 \mathrm{H}, \mathrm{d}, J 1.5) ; \delta_{\mathrm{C}}\left(\mathrm{CDCl}_{3}, 100 \mathrm{MHz}\right) 24.90(\mathrm{q}), 27.80(\mathrm{q})$, 30.20 (t), $31.00(\mathrm{t}), 35.06(\mathrm{~d}), 44.70$ (t), 47.60 (d), 51.86 (d), 59.90 (q), 73.10 (d), 77.82 (d), 100.76 (t), 107.91 (d), 108.64 (d), 108.82 (s), 122.29 (d), 133.51 (s), $146.30(\mathrm{~s}), 147.40$ (s), 155.46 (s); $m / z$ (EI) 375 (15), 360 (10), 317 (100), 299 (50), 545242 (10), 215 (15), 190 (10), 177 (15), 140 (100), 126 (10), 88 (10); Found: $\mathrm{m} / z \mathrm{M}^{+}$, $375.1682, \mathrm{C}_{20} \mathrm{H}_{25} \mathrm{NO}_{6}$, requires $375.1675(\Delta=+1.9 \mathrm{ppm})$.

$(3 \mathrm{a} R, 5 S, 5 \mathrm{a} R, 11 \mathrm{~b} S, 11 \mathrm{c} R)-5,5 \mathrm{a}-$ Dihydroxyisopropylidene-1,2,3b,3a,4,5,11b,11coctahydro-[1,3]dioxolo[4,5-f]pyrrolo-[3,2,1-de]phenantridin-7-one 19 (Using the Banwell modification of Bischler-Napieralski $\left.{ }^{49},{ }^{50}\right)$ : To a stirred solution of 550 methyl carbamate $(+)-18(100 \mathrm{mg}, 0.266 \mathrm{mmol})$ and 4-DMAP $(98 \mathrm{mg}, 0.80 \mathrm{mmol})$ in $\mathrm{CH}_{2} \mathrm{Cl}_{2}(10 \mathrm{~mL})$ at $0{ }^{\circ} \mathrm{C}$ was added dropwise trifluoromethylsulfonic anhydride ( $251 \mu \mathrm{L}, 376 \mathrm{mg} .1 .33 \mathrm{mmol}$ ). The resulting dark suspension was allowed to warm to $\mathrm{RT}$ and stir for a further $14 \mathrm{~h}$. The reaction mixture was then diluted with $\mathrm{CH}_{2} \mathrm{Cl}_{2}$ $(40 \mathrm{~mL})$, washed with sat. aq. $\mathrm{NaHCO}_{3}(20 \mathrm{~mL})$, the aqueous phase extracted with $555 \mathrm{CH}_{2} \mathrm{Cl}_{2}(3 \times 30 \mathrm{~mL})$, the combined organic phases washed with sat. aq. $\mathrm{NH}_{4} \mathrm{Cl}(20$ $\mathrm{mL})$, the aqueous phase extracted with $\mathrm{CH}_{2} \mathrm{Cl}_{2}(30 \mathrm{~mL})$, the combined organic phases dried over $\mathrm{Na}_{2} \mathrm{SO}_{4}$ and concentrated in vacuo. The residue was purified by $\mathrm{FC}\left[\mathrm{SiO}_{2}, \mathrm{Et}_{2} \mathrm{O} /\right.$ pentane/ $\mathrm{MeOH}$ ( $\mathrm{NH}_{3}$ sat.), 60:35:5] to give lactam (-)-19 as a white solid $(76 \mathrm{mg}, 82 \%)$ : Mp $224{ }^{\circ} \mathrm{C}\left(\mathrm{Et}_{2} \mathrm{O} /\right.$ pentane); $[\alpha]_{\mathrm{D}}-75.4$ (c. $\left.0.92, \mathrm{CHCl}_{3}\right)$; $560 v_{\max } / \mathrm{cm}^{-1}\left(\mathrm{CHCl}_{3}\right) 2928,1642,1609,1504,1461.1415,1384,1352,1270,1241$, $1211,1163,1127,1043,937,874,832 ; \delta_{\mathrm{H}}\left(\mathrm{CDCl}_{3}, 400 \mathrm{MHz}\right) 1.44(3 \mathrm{H}, \mathrm{s}), 1.53$ $(3 \mathrm{H}, \mathrm{s}), 1.67(2 \mathrm{H}, \mathrm{m}), 2.24(2 \mathrm{H}, \mathrm{m}), 2.43(1 \mathrm{H}, \mathrm{m}), 2.96(1 \mathrm{H}, \mathrm{dd}, J 14.5,8), 3.23(1 \mathrm{H}$, dt, $J 12,5.5), 3.52(1 \mathrm{H}, \mathrm{dd}, J 14,10.5), 4.22(1 \mathrm{H}, \mathrm{dd}, J 12,5.5), 4.30(2 \mathrm{H}, \mathrm{m}), 6.01$ $\left(2 \mathrm{H}, \mathrm{AB}_{\mathrm{q}}, J 1.5\right), 7.19(1 \mathrm{H}, \mathrm{d}, J 1), 7.49(1 \mathrm{H}, \mathrm{s}) ; \delta_{\mathrm{C}}\left(\mathrm{CDCl}_{3}, 100 \mathrm{MHz}\right) 24.44(\mathrm{q})$, 56527.25 (q), 32.20 (t), $32.41(\mathrm{t}), 34.95$ (d), 40.59 (d), 45.16 (t), 57.32 (d), 74.89 (d), 75.69 (d), 101.48 (t), 106.29 (d), 108.25 (d), 109.52 (s), 124.76 (s), 135.33 (s), 146.88 (s), 150.61 (s), 161.91 (s); $\mathrm{m} / \mathrm{z}$ (EI) 344 (7), 343 (10), 268 (6), 121 (26), 111 (18), 97 (29), 85 (27), 69 (56), 57 (100); Found: $m / z \mathrm{M}^{+}, 343.1415, \mathrm{C}_{19} \mathrm{H}_{21} \mathrm{NO}_{5}$, requires $343.1419(\Delta=-1.2 \mathrm{ppm})$

$570(\mathbf{3 a} R, 5 S, 5 \mathrm{a} R, \mathbf{1 1 b} S, \mathbf{1 1} \mathrm{c} R)-\mathbf{5 , 5 a}$-Dihydroxyisopropylidene-1,2,3b,3a,4,5,11b,11coctahydro-[1,3]dioxolo[4,5-f]pyrrolo-[3,2,1-de]phenantridine 20.

Method 1 [ $\mathrm{LiAlH}_{4}$ reduction of lactam (-)-19]: To a solution of lactam (-)-19 (12 $\mathrm{mg}, 0.03 \mathrm{mmol})$ in THF $(3 \mathrm{~mL})$ at RT was added $\mathrm{LiAlH}_{4}(4 \mathrm{mg}, 0.11 \mathrm{mmol})$. After stirring for $2 \mathrm{~h}$, the reaction mixture was quenched with brine $(3 \mathrm{~mL})$ and 575 concentrated in vacuo. The residue was partitioned between $\mathrm{CH}_{2} \mathrm{Cl}_{2}(5 \mathrm{~mL})$ and $\mathrm{H}_{2} \mathrm{O}$ $(3 \mathrm{~mL})$ and the organic phase was extracted with $\mathrm{CH}_{2} \mathrm{Cl}_{2}(3 \times 5 \mathrm{~mL})$. The combined organic phases were dried over $\mathrm{Na}_{2} \mathrm{CO}_{3}$ and concentrated in vacuo to give amine (-)20 as white solid $(11 \mathrm{mg}, 95 \%)$ : $\mathrm{Mp} 162-164{ }^{\circ} \mathrm{C}\left(\mathrm{Et}_{2} \mathrm{O} /\right.$ pentane) [NB. sublimes 155 $\left.{ }^{\circ} \mathrm{C}\right] ; \mathrm{R}_{\mathrm{f}} 0.35$ (EtOAc/MeOH, 4:1); $[\alpha]_{\mathrm{D}}-14.0\left(\right.$ c. $\left.0.50, \mathrm{CHCl}_{3}\right) ; v_{\max } / \mathrm{cm}^{-1}(\mathrm{KBr}) 3440$, $5802988,2905,2775,1485,1380,1252,1237,1050,1036,933 ; \delta_{\mathrm{H}}\left(\mathrm{CDCl}_{3}, 400 \mathrm{MHz}\right)$ $1.40(3 \mathrm{H}, \mathrm{s}), 1.53(3 \mathrm{H}, \mathrm{s}), 1.55-1.68(2 \mathrm{H}), 2.11(3 \mathrm{H}, \mathrm{m}), 2.27(1 \mathrm{H} \mathrm{t}, J 10), 2.47(1 \mathrm{H}$, td, $J$ 9, 5), $2.84(1 \mathrm{H}, \mathrm{t}, J 10), 3.07$ (1H, ddd, $J 10,6,2), 3.70(1 \mathrm{H}, \mathrm{dd}, J 14.5,1), 4.01$ $(1 \mathrm{H}, \mathrm{d}, J 14.5), 4.14(1 \mathrm{H}, \mathrm{dd}, J 10,7.5), 4.24(1 \mathrm{H}, \mathrm{ddd}, J 11,7.5,5), 5.91(2 \mathrm{H}, \mathrm{s})$, $6.55(1 \mathrm{H}, \mathrm{s}), 7.31(1 \mathrm{H}, \mathrm{s}) ; \delta_{\mathrm{C}}\left(\mathrm{CDCl}_{3}, 100 \mathrm{MHz}\right) 24.2(\mathrm{q}), 27.2(\mathrm{q}), 31.8(\mathrm{t}), 32.2(\mathrm{t})$, $58533.2(\mathrm{~d}), 41.4(\mathrm{~d}), 53.6(\mathrm{t}), 55.7(\mathrm{t}), 62.1(\mathrm{~d}), 75.5(\mathrm{~d}), 76.6(\mathrm{~d}), 100.7(\mathrm{t}), 106.5(\mathrm{~d})$, $107.8(\mathrm{~d}), 108.7(\mathrm{~s}), 129.1(\mathrm{~s}), 132.2(\mathrm{~s}), 145.9(\mathrm{~s}), 146.1(\mathrm{~s}) ; \mathrm{m} / \mathrm{z}\left(\mathrm{CI}^{+}\right) 330\left(\mathrm{MH}^{+}\right.$, 100); $m / z\left(\mathrm{EI}^{+}\right) 329$ ( $\left.\mathrm{M}^{+}, 44\right), 328$ (100), 314 (7), 270 (4), 254 (6), 214 (2), 187 (3); Found: $m / z \mathrm{M}^{+}, 329.1619, \mathrm{C}_{19} \mathrm{H}_{23} \mathrm{NO}_{4}$, requires $329.1627(\Delta=-2.4 \mathrm{ppm})$. For a single crystal X-ray structure determination on this compound see ESI.

590 Crystal data for 20: $\mathrm{C}_{19} \mathrm{H}_{23} \mathrm{NO}_{4}, \mathrm{M}=329.38$, hexagonal, $\mathrm{P} 6$ ( 10.695(1), c = 25.134(4) $\AA, \mathrm{V}=2489.7(5) \AA^{3}, \mathrm{Z}=6, \mathrm{D}_{\mathrm{c}}=1.318 \mathrm{~g} \mathrm{~cm}^{-3}, \mu(\mathrm{Mo}-\mathrm{K} \alpha)$ $=0.092 \mathrm{~mm}^{-1}, \mathrm{~T}=298 \mathrm{~K}$, colourless prisms, Stoe Stadi-4 diffractometer; 2331 independent measured reflections $\left(R_{\text {int }}=0.0\right), F^{2}$ refinement, $R_{1}(o b s)=0.0446$, $\mathrm{wR}_{2}$ (all) $=0.0846,1415$ independent observed absorption-corrected reflections $\left[\left|\mathrm{F}_{\mathrm{o}}\right|\right.$

$\left.595>4 \sigma\left(\left|\mathrm{F}_{\mathrm{o}}\right|\right), 2 \theta_{\max }=46^{\circ}\right], 218$ parameters. The absolute structure of 20 could not be determined by either an R-factor test $\left[R_{1}^{+}=0.0446, R_{1}^{-}=0.0446\right]$ or by use of the Flack parameter $\left[\mathrm{x}^{+}=0.0(17), \mathrm{x}^{-}=2.6(17)\right]$, and so was assigned by internal reference on $\mathrm{C}(7), \mathrm{C}(8), \mathrm{C}(12), \mathrm{C}(14)$ and $\mathrm{C}(22)$. CCDC 792818 .

Method 2 [Pictet-Spengler reaction ${ }^{46,47}$ using Eschenmoser's salt on hydrindane $600(+)-6$ ]: To a solution of amine (+)-6 $(173 \mathrm{mg}, 0.54 \mathrm{mmol})$ in THF $(20 \mathrm{~mL})$ was added Eschenmoser's salt ${ }^{66}(151 \mathrm{mg}, 0.82 \mathrm{mmol}, 1.5$ equiv $)$ and the suspension stirred at $40{ }^{\circ} \mathrm{C}$. After $40 \mathrm{~h}$ the solution was quenched with $\mathrm{NH}_{4} \mathrm{OH}(10 \%$ aq. 10 $\mathrm{mL}$ ) and the resulting biphasic solution concentrated in vacuo. The residue was suspended in $\mathrm{CH}_{2} \mathrm{Cl}_{2}(30 \mathrm{~mL})$, washed with water $(10 \mathrm{~mL})$, dried over $\mathrm{MgSO}_{4}$ and 605 concentrated in vacuo. The residue was purified by $\mathrm{FC}\left(\mathrm{Et}_{2} \mathrm{O} /\right.$ pentane, $1: 1 \rightarrow \mathrm{Et}_{2} \mathrm{O}$; column presaturated with $\left.\mathrm{Et}_{3} \mathrm{~N} / \mathrm{Et}_{2} \mathrm{O}, 1: 9\right)$ to give amine (-)-20 as a white solid (95.2 $\mathrm{mg}, 53 \%)$ and recovered amine (+)-6 (12.0 mg, 7\%). Spectroscopic data as above. 
(3a $R, 5 S, 6 R, 7 S, 7 \mathrm{a} R)-5,6-\mathrm{Di}-O \cdot$ isopropylidene-1-phenoxycarbonyl-7-[3,4(methylenedioxy)-6-chloromethylphenyl]-2,3,3a,4,5,6,7,7a-octahydroindole 21 .

610 To a stirred suspension of amine (-)-20 (3.2 $\mathrm{mg}, 0.040 \mathrm{mmol})$ and $\mathrm{NaHCO}_{3}(50 \mathrm{mg})$ in $\mathrm{CH}_{2} \mathrm{Cl}_{2}(5 \mathrm{~mL})$ at $0{ }^{\circ} \mathrm{C}$ was added a solution of phenylchloroformate in $\mathrm{CH}_{2} \mathrm{Cl}_{2}$ ( $70 \mu \mathrm{L}, 1 \mathrm{M}$ ) dropwise. The suspension was stirred for $2 \mathrm{~h}$ at $0{ }^{\circ} \mathrm{C}$ and then refluxed for $13 \mathrm{~h}$. The reaction mixture was then diluted with $\mathrm{CH}_{2} \mathrm{Cl}_{2}(30 \mathrm{~mL})$, washed with aq. sat. $\mathrm{NaHCO}_{3}$, the aqueous phase extracted $\mathrm{CH}_{2} \mathrm{Cl}_{2}(4 \times 10 \mathrm{~mL})$, the combined 615 organic phases dried over $\mathrm{Na}_{2} \mathrm{SO}_{4}$ and concentrated in vacuo. The residue was purified by $\mathrm{FC}\left(\mathrm{SiO}_{2} ; \mathrm{Et}_{2} \mathrm{O} /\right.$ pentane, 1:1) to give phenyl carbamate (-)-21 as a yellow waxy solid (17.4 mg, 89\%): $\mathrm{Mp} 64{ }^{\circ} \mathrm{C}\left(\mathrm{Et}_{2} \mathrm{O} /\right.$ pentane $) ;[\alpha]_{\mathrm{D}}-24.3\left(\right.$ c. $\left.0.42, \mathrm{CHCl}_{3}\right)$; $v_{\max } / \mathrm{cm}^{-1}\left(\mathrm{CHCl}_{3}\right) 3020,1709,1507,1488,1406,1220,1073,1043,929,792 ; \delta_{\mathrm{H}}$ $\left(\mathrm{CDCl}_{3}, 400 \mathrm{MHz}, 328 \mathrm{~K}\right) 1.30(3 \mathrm{H}, \mathrm{s}), 1.49(3 \mathrm{H}, \mathrm{s}), 1.93(2 \mathrm{H}, \mathrm{m}), 2.20(2 \mathrm{H}, \mathrm{m})$,

$6202.54(1 \mathrm{H}, \mathrm{m}), 3.31(1 \mathrm{H}, \mathrm{t}, J 10.5), 3.53(1 \mathrm{H}, \mathrm{m}), 3.82(1 \mathrm{H}, \mathrm{ddd}, J 8,8,5.5), 4.33$ $(3 \mathrm{H}, \mathrm{m}), 4.62\left(2 \mathrm{H}, \mathrm{AB}_{\mathrm{q}}, J 11.5\right), 5.54(1 \mathrm{H}$, broad s $), 5.81(1 \mathrm{H}$, broad s $), 6.74(2 \mathrm{H}, \mathrm{d}$, $J 7), 6.75(1 \mathrm{H}, \mathrm{s}), 6.94(1 \mathrm{H}, \mathrm{s}), 7.07(1 \mathrm{H}, \mathrm{t}, J 7.5), 7.22(2 \mathrm{H}, \mathrm{t}, J 7.5) ; \delta_{\mathrm{C}}\left(\mathrm{CDCl}_{3}, 100\right.$ MHz, 328 K, 1 carbon absent) 24.69 (q), 27.65 (q), 30.77 (t), 35.47 (d), 42.63 (d), $44.58(\mathrm{t}), 45.51(\mathrm{t}), 60.23$ (d), 73.19 (d), 79.37 (d), 101.17 (t), $108.81(\mathrm{~s}), 108.81$ (d), 625109.87 (d), $121.10(2 \times \mathrm{d}), 124.59$ (d), $128.83(2 \times \mathrm{d}), 130.35$ (s), $133.46(\mathrm{~s}), 146.38$ (s), 148.44 (s), 151.44 (s), 153.05 (s); m/z (EI) 487 (35), 485 (100), 450 (80), 427 (90), 392 (30), 334 (100), 298 (90), 202 (75), 97 (50), 71 (60); Found: $\mathrm{m} / \mathrm{z} \mathrm{M}^{+}$, $485.1614, \mathrm{C}_{26} \mathrm{H}_{28} \mathrm{NO}_{6}{ }^{35} \mathrm{Cl}$, requires $485.1598(\Delta=+3.3 \mathrm{ppm})$.

$(3 \mathrm{a} R, 5 S, 6 R, 7 S, 7 \mathrm{a} R)-5,6-\mathrm{Di}-O$-isopropylidene-1-phenoxycarbonyl-7-[3,4630 (methylenedioxy)-6-oxomethylphenyl]-2,3,3a,4,5,6,7,7a-octahydroindole 22 . To a stirred solution of benzylchloride (-)-21 (10 mg, $0.0206 \mathrm{mmol})$ in DMSO (2 mL) was added trimethylamine- $N$-oxide $(50 \mathrm{mg}, 0.533 \mathrm{mmol})$ and the resulting solution stirred at RT for $24 \mathrm{~h}$. The reaction mixture was then diluted with $\mathrm{Et}_{2} \mathrm{O}(20 \mathrm{~mL})$ and partitioned with sat. aq. $\mathrm{NH}_{4} \mathrm{Cl}(20 \mathrm{~mL})$. The phases were separated, the aqueous 635 phase was extracted with $\mathrm{Et}_{2} \mathrm{O}(3 \times 20 \mathrm{~mL})$ and the combined organic phases dried over $\mathrm{Na}_{2} \mathrm{SO}_{4}$ and concentrated in vacuo. The residue was purified by $\mathrm{FC}\left(\mathrm{SiO}_{2}\right.$ $\mathrm{Et}_{2} \mathrm{O} /$ pentane, 9:1) to give aldehyde (+)-22 as a white solid $(7.8 \mathrm{mg}, 81 \%)$ : Mp 74 ${ }^{\circ} \mathrm{C}\left(\mathrm{Et}_{2} \mathrm{O} /\right.$ pentane $) ;[\alpha]_{\mathrm{D}}+20.4\left(c .0 .35, \mathrm{CHCl}_{3}\right) ; v_{\max } / \mathrm{cm}^{-1}\left(\mathrm{CHCl}_{3}\right) 3022,2926$, $1712,1612,1505,1486,1384,1252,1199,1043,937,867,770,730 ; \delta_{\mathrm{H}}\left(\mathrm{CDCl}_{3}\right.$, $640400 \mathrm{MHz}, 331 \mathrm{~K}) 1.32(3 \mathrm{H}, \mathrm{s}), 1.46(3 \mathrm{H}, \mathrm{s}), 2.23(4 \mathrm{H}, \mathrm{m}), 2.51(1 \mathrm{H}, \mathrm{d}, J$ 7.5), 3.50 $(1 \mathrm{H}, \mathrm{m}), 3.73(1 \mathrm{H}, \mathrm{td}, J 11.5,7.5), 4.16(1 \mathrm{H}, \mathrm{m}), 4.30(1 \mathrm{H}, \mathrm{m}), 4.40(1 \mathrm{H}, \mathrm{m}), 4.48$ $(1 \mathrm{H}, \mathrm{m}), 5.69(1 \mathrm{H}$, broad s), $5.92(1 \mathrm{H}, \mathrm{s}), 6.69(2 \mathrm{H}, \mathrm{m}), 7.08(2 \mathrm{H}, \mathrm{m}), 7.22(3 \mathrm{H}, \mathrm{m})$, $10.10(1 \mathrm{H}, \mathrm{s}) ; \delta_{\mathrm{C}}\left(\mathrm{CDCl}_{3}, 100 \mathrm{MHz}, 331 \mathrm{~K}, 1\right.$ carbon absent) 25.09 (q), 27.71 (q), 29.69 (t), $30.04(\mathrm{t}), 31.18$ (t), 35.69 (d), 39.96 (d), 45.82 (t), 61.00 (d), 73.34 (d),

64578.00 (d), $101.85(\mathrm{t}), 108.48(2 \times \mathrm{d}), 109.13(2 \times \mathrm{d}), 120.95$ (d), $124.71(\mathrm{~d}), 128.91$ (s), 130.80 (s), 139.69 (s), 147.22 (s), 151.31 (s), 152.90 (s), 189.66 (d); m/z (EI) 465 (20), 450 (10), 407 (25), 344 (100), 268 (30), 205 (25), 175 (100), 77 (20). Found: $m / z \mathrm{M}^{+}, 465.1788, \mathrm{C}_{26} \mathrm{H}_{27} \mathrm{NO}_{7}$, requires $465.1780(\Delta=+1.7 \mathrm{ppm})$.

(3a $R, 5 S, 6 R, 7 S, 7 \mathrm{a} R)$-5,6-Di- $O$-isopropyfidene-l-methyl-7-[3,4-(methylendioxy)-6650 hydroxymethylphenyl]-2,3,3a,4,5,6,7,7a-octahydroindole 23 . To a solution of aldehyde (+)-22 $(55 \mathrm{mg}, 0.118 \mathrm{mmol})$ in THF $(8 \mathrm{~mL})$ was added a solution of $\mathrm{LiAlH}_{4}(64 \mathrm{mg}, 1.88 \mathrm{mmol})$ in $\mathrm{Et}_{2} \mathrm{O}(12 \mathrm{~mL})$ and the reaction mixture heated at reflux for $4.5 \mathrm{~h}$. The reaction mixture was cooled to $\mathrm{RT}$ and $\mathrm{MeOH}(1 \mathrm{~mL})$ added dropwise. The reaction mixture was then partitioned between $\mathrm{CH}_{2} \mathrm{Cl}_{2}(25 \mathrm{~mL})$ and

655 sat. brine $(25 \mathrm{~mL})$. The phases were separated, the aqueous phase extracted with $\mathrm{CH}_{2} \mathrm{Cl}_{2}(5 \times 20 \mathrm{~mL})$, the combined organic phases dried over $\mathrm{Na}_{2} \mathrm{SO}_{4}$ and concentrated in vacuo. The residue was purified by $\mathrm{FC}\left(\mathrm{SiO}_{2} ; \mathrm{Et}_{2} \mathrm{O} /\right.$ pentane/MeOH $\left(\mathrm{NH}_{3}\right.$ sat.), 58:40:2] to give benzyl alcohol (+)-23 as a colourless oil (38 $\left.\mathrm{mg}, 89 \%\right)$ : $[\alpha]_{\mathrm{D}}+15.4\left(c .1 .14, \mathrm{CHCl}_{3}\right) ; v_{\max } / \mathrm{cm}^{-1}\left(\mathrm{CHCl}_{3}\right) 3521,2987,2889,1616,1502,1480$,

$6601458,1382,1262,1229,1077,1044,930,870,793,750 ; \delta_{\mathrm{H}}\left(\mathrm{CDCl}_{3}, 400 \mathrm{MHz}\right) 1.31$ $(3 \mathrm{H}, \mathrm{s}), 1.42(3 \mathrm{H}, \mathrm{s}), 1.64(5 \mathrm{H}$, broad s $), 2.06(1 \mathrm{H}, \mathrm{m}), 2.17(1 \mathrm{H}, \mathrm{td}, \mathrm{J} 13,5.5), 2.32$ $(3 \mathrm{H}, \mathrm{m}), 3.08(1 \mathrm{H}, \mathrm{m}), 3.21(1 \mathrm{H}, \mathrm{t}, J 10.5), 4.23(1 \mathrm{H}, \mathrm{d}, J 12), 4.31(1 \mathrm{H}, \mathrm{m}), 4.43$ $(1 \mathrm{H}, \mathrm{dd}, J 10,7.5), 4.82(1 \mathrm{H}, \mathrm{d}, J 11.5), 5.95\left(2 \mathrm{H}, \mathrm{AB}_{\mathrm{q}}, J 1\right), 6.85(1 \mathrm{H}, \mathrm{s}), 6.89(1 \mathrm{H}$, $\mathrm{s}) ; \delta_{\mathrm{C}}\left(\mathrm{CDCl}_{3}, 100 \mathrm{MHz}\right) 24.35(\mathrm{q}), 27.29(\mathrm{q}), 32.07(\mathrm{t}), 32.53(\mathrm{t}), 36.28(\mathrm{~d}), 43.16$ $665(\mathrm{~d}), 43.74(\mathrm{~d}), 56.98(\mathrm{t}), 63.18(\mathrm{t}), 68.47(\mathrm{q}), 74.23(\mathrm{~d}), 77.86(\mathrm{~d}), 101.11(\mathrm{t}), 106.05$ $(2 \times d), 108.85(2 \times d), 111.02$ (d), 132.89 (s), 135.46 (s), 146.15 (s), 147.97 (s).

(3aR,5S,6R,7S,7aR)-5,6-Di- $O$-isopropylidene-l-methyl-7-[3,4-(methylenedioxy)6-oxomethylphenyl]-2,3,3a,4.5,6,7,7a-octahydroindole 24 . To a stirred solution of benzyl alcohol (+)-23 $(24 \mathrm{mg}, 0.066 \mathrm{mmol})$ in $\mathrm{CH}_{2} \mathrm{Cl}_{2}(8 \mathrm{~mL})$ at $\mathrm{RT}$ was added a 670 solution of Dess-Martin periodinane $(35 \mathrm{mg}, 0.083 \mathrm{mmol})$ in $\mathrm{CH}_{2} \mathrm{Cl}_{2}(7 \mathrm{~mL})$ dropwise. After $75 \mathrm{~min}$ a further portion of periodinane $(20 \mathrm{mg}, 0.047 \mathrm{mmol})$ was added and the reaction mixture was stirred for further $2.5 \mathrm{~h}$ at RT. The reaction mixture was then diluted with $\mathrm{CH}_{2} \mathrm{Cl}_{2}(30 \mathrm{~mL})$, washed with sat. aq. $\mathrm{NaHCO}_{3}(20$ $\mathrm{mL})$, the phases separated, the aqueous phase reextracted with $\mathrm{CH}_{2} \mathrm{Cl}_{2}(3 \times 10 \mathrm{~mL})$,

675 the combined organic phases dried over $\mathrm{Na}_{2} \mathrm{SO}_{4}$ and the solvent evaporated in vacuo. The residue was purified by $\left(\mathrm{SiO}_{2} ; \mathrm{Et}_{2} \mathrm{O} /\right.$ pentane/MeOH $\left(\mathrm{NH}_{3}\right.$ sat.), 60:37:3] to give aldehyde (+)-24 as a pale yellow solid $(8.8 \mathrm{mg}, 37 \%): v_{\max } / \mathrm{cm}^{-1}\left(\mathrm{CHCl}_{3}\right)$ 2921, 2780, 1671, 1616, 1480, 1458, 1382, 1365, 1284, 1251, 1158, 1044, 935, 864,
$641 ; \delta_{\mathrm{H}}\left(\mathrm{CDCl}_{3}, 200 \mathrm{MHz}\right) 1.29(3 \mathrm{H}, \mathrm{s}), 1.40(3 \mathrm{H}, \mathrm{s}), 1.57(1 \mathrm{H}, \mathrm{m}), 1.72(3 \mathrm{H}, \mathrm{s})$, $6802.11(1 \mathrm{H}, \mathrm{m}), 2.97(1 \mathrm{H}, \mathrm{dd}, J 8,7.5), 3.71(1 \mathrm{H}, \mathrm{t}, J 9), 4.28(2 \mathrm{H}, \mathrm{m}), 6.01\left(2 \mathrm{H}, \mathrm{AB}_{\mathrm{q}}\right.$, $J$ 1), $6.94(1 \mathrm{H}, \mathrm{s}), 7.40(1 \mathrm{H}, \mathrm{s}), 10.26(1 \mathrm{H}, \mathrm{s}) ; \delta_{\mathrm{C}}\left(\mathrm{CDCl}_{3}, 50 \mathrm{MHz}\right.$, diagnostic peaks only) 24.21 (q), 27.12 (q), 31.15 (d), 32.74 (t), 35.78 (t), 41.29 (d), 42.32 (d), 57.11 (t), 67.89 (q), 74.16 (d), 78.25 (d), $101.82(\mathrm{t}), 106.12(\mathrm{~d}), 107.30$ (d).

$\left(3 \mathrm{a} R^{*}, 5 S^{*}, 6 R^{*}, 7 S^{*}, 7 \mathrm{a} R^{*}\right)-5,6-\mathrm{Di}-O$-isopropylidene-1-phenylcarbamate-7-[3,4685 (methylenedioxy)phenyl]-2,3,3a,4,5,6,7,7a-octahydroindole 25 . To a solution of pyrrolidine ( \pm )-6 $(620 \mathrm{mg}, 1.96 \mathrm{mmol}), \mathrm{Et}_{3} \mathrm{~N}(1 \mathrm{~mL}, 7.82 \mathrm{mmol})$ and DMAP (17 $\mathrm{mg}, 0.02 \mathrm{mmol})$ in $\mathrm{CH}_{2} \mathrm{Cl}_{2}(10 \mathrm{~mL})$ at $\mathrm{RT}$ was added phenyl chloroformate $(736 \mu \mathrm{L}$, $5.87 \mathrm{mmol}$ ). After stirring for $10 \mathrm{~min}$, the reaction mixture was quenched with $\mathrm{NaHCO}_{3}$ (sat. aq., $\left.5 \mathrm{~mL}\right)$ and the organic phase was extracted with $\mathrm{CH}_{2} \mathrm{Cl}_{2}(3 \times 10$ $690 \mathrm{~mL}$ ). The combined organic phases were dried over $\mathrm{Na}_{2} \mathrm{SO}_{4}$, filtered and concentrated in vacuo. The residue was purified by $\mathrm{FC}\left(\mathrm{SiO}_{2}\right.$; petrol/Et $\left.\mathrm{Et}_{2} \mathrm{O}, 1: 1\right)$ to give carbamate $( \pm)-25$ as a white solid $(670 \mathrm{mg}, 78 \%)$ : $\mathrm{Mp} 231.5-234.7^{\circ} \mathrm{C} . \mathrm{R}_{f} 0.30$

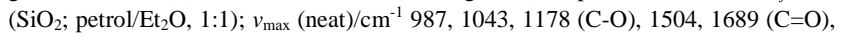
2861, 2942; $\delta_{\mathrm{H}}\left(\mathrm{CDCl}_{3}, 500 \mathrm{MHz}, 328 \mathrm{~K}\right) 1.35\left(3 \mathrm{H}, \mathrm{s}, \mathrm{CH}_{3}\right), 1.50\left(3 \mathrm{H}, \mathrm{s}, \mathrm{CH}_{3}\right), 1.89$ $695\left(1 \mathrm{H}, \mathrm{m}, \mathrm{C}_{4} H \mathrm{H}\right), 1.99\left(1 \mathrm{H}, \mathrm{m}, \mathrm{C}_{3} H \mathrm{H}\right), 2.18-2.24\left(2 \mathrm{H}, \mathrm{C}_{4} \mathrm{H} H \& \mathrm{C}_{3} H \mathrm{H}\right), 2.54(1 \mathrm{H}, a p p$ sext, $\left.J 7, \mathrm{C}_{3 \mathrm{a}} \mathrm{H}\right), 2.78\left(1 \mathrm{H}, \mathrm{dd}, J 11.5,10.5, \mathrm{C}_{11 \mathrm{~b}} \mathrm{H}\right), 3.48\left(1 \mathrm{H}, \mathrm{m}, \mathrm{C}_{2} H \mathrm{H}\right), 3.81(1 \mathrm{H}$, $\left.\mathrm{m}, \mathrm{C}_{2} \mathrm{HH}\right), 4.19\left(1 \mathrm{H}, \mathrm{m}, \mathrm{C}_{11 \mathrm{c}} \mathrm{H}\right), 4.35\left(1 \mathrm{H}, \mathrm{td}, J 8.5,6.0, \mathrm{C}_{5} \mathrm{H}\right), 4.48\left(1 \mathrm{H}, \mathrm{m}, \mathrm{C}_{5 \mathrm{a}} \mathrm{H}\right)$, $5.71\left(1 \mathrm{H}\right.$, br s, OCHHO), $5.85(1 \mathrm{H}$, br s, OCHHO $), 6.65-6.75\left(3 \mathrm{H}, 3 \times \mathrm{C}_{\mathrm{ar}} \mathrm{H}\right), 6.81$ $\left(1 \mathrm{H}, \mathrm{d}, J 1.5, \mathrm{C}_{\mathrm{ar}} \mathrm{H}\right), 7.09\left(1 \mathrm{H}, \mathrm{t}, J 7.0, \mathrm{C}_{\mathrm{ar}} \mathrm{H}\right), 7.23\left(2 \mathrm{H}, \mathrm{t}, J 7.0, \mathrm{C}_{\mathrm{ar}} \mathrm{H}\right), 7.28(1 \mathrm{H}$,

$\left.700 \mathrm{C}_{\mathrm{ar}} \mathrm{H}\right) ; \delta_{\mathrm{C}}\left(\mathrm{CDCl}_{3}, 125 \mathrm{MHz}, 328 \mathrm{~K}\right) 24.95(\mathrm{q}), 27.85(\mathrm{q}), 29.85(\mathrm{t}), 30.63(\mathrm{t}), 34.63$ (d), $45.01(\mathrm{t}), 47.52$ (d), 60.42 (d), $73.74(\mathrm{~d}), 78.68$ (d), 100.82 (t), 108.13 (d), 108.49 (d), 108.75 (s), 120.99 ( $2 \times$ d), 122.75 (d), 124.74 (d), $129.28(2 \times$ d), 133.09 (s), 146.65 (s), 147.79 (s), 151.02 (s), 153.07 (s); m/z (CI') $438\left(\mathrm{MNH}^{+}, 90\right), 455$ $\left(\mathrm{MNH}_{4}{ }^{+}, 100\right)$; Found: $m / z \mathrm{MH}^{+}, 438.1920, \mathrm{C}_{25} \mathrm{H}_{28} \mathrm{NO}_{6}$, requires $438.1917(\Delta=$ $705+0.8$ ); Calculated for $\mathrm{C}_{25} \mathrm{H}_{27} \mathrm{NO}_{6} \mathrm{C}, 68.63 \% ; \mathrm{H}, 6.22 \%$; N, 3.20\%; O, 21.94\%, found $\mathrm{C}, 68.60 \% ; \mathrm{H}, 6.21 \% ; \mathrm{N}, 3.18 \% ; \mathrm{O}, 22.01 \%$.

\section{$\left(3 \mathrm{a} R *, 5 S^{*}, 6 R^{*}, 7 S^{*}, 7 \mathrm{a} R *\right)-5,6-\mathrm{Di}-O$-isopropylidene-1-methyl-7-[3,4-} (methylenedioxy)phenyl]-2,3,3a,4,5,6,7,7a-octahydroindole 26. To a solution of phenyl carbamate $( \pm)-25(199 \mathrm{mg}, 0.45 \mathrm{mmol})$ in THF $(20 \mathrm{~mL})$ at RT was added $710 \mathrm{LiAlH}_{4}(258 \mathrm{mg}, 0.68 \mathrm{mmol})$. The resulting suspension was refluxed in the dark for $3 \mathrm{~h}$ and then allowed to cool to RT. The reaction mixture was then partitioned between brine $(10 \mathrm{~mL})$ and EtOAc $(15 \mathrm{~mL})$ and the organic phase was extracted with EtOAc $(3 \times 15 \mathrm{~mL})$. The combined organic phases were dried over $\mathrm{Na}_{2} \mathrm{CO}_{3}$, filtered and concentrated in vacuo to afford $N$-methylindoline ( \pm )-26 as a colourless 715 oil (130 mg, 88\%). $\mathrm{R}_{f} 0.35\left(\mathrm{SiO}_{2}\right.$; petrol/Et $\left.2 \mathrm{O}, 1: 4\right) ; v_{\max }($ neat $) / \mathrm{cm}^{-1} 983,1050,1187$, $1504,2875,2942 ; \delta_{\mathrm{H}}\left(\mathrm{CDCl}_{3}, 400 \mathrm{MHz}\right) 1.30\left(3 \mathrm{H}, \mathrm{s}, \mathrm{CH}_{3}\right), 1.46\left(3 \mathrm{H}, \mathrm{s}, \mathrm{CH}_{3}\right), 1.53$ $\left(1 \mathrm{H}, \mathrm{ddd}, J 12.0,10.0,6.0, \mathrm{C}_{4} \mathrm{H} H\right), 1.68\left(1 \mathrm{H}, \mathrm{dt}, J 13.0,10.5, \mathrm{C}_{3} H \mathrm{H}\right), 1.82(3 \mathrm{H}, \mathrm{s}$, $\left.\mathrm{NCH}_{3}\right), 2.03\left(1 \mathrm{H}\right.$, ddd, $J$ 12.0, 8.5, 6.0, $\left.\mathrm{C}_{4} H \mathrm{H}\right), 2.13-2.25\left(2 \mathrm{H}, \mathrm{C}_{11 \mathrm{~b}} \mathrm{H}, \mathrm{C}_{3} \mathrm{H} H\right), 2.25$ $\left(1 \mathrm{H}, \mathrm{m}, \mathrm{C}_{3 \mathrm{a}} \mathrm{H}\right), 2.32\left(1 \mathrm{H}, \mathrm{t}, J 10.0, \mathrm{C}_{2} \mathrm{H} H\right), 2.72\left(1 \mathrm{H}, \mathrm{t}, J 10.0, \mathrm{C}_{2} H \mathrm{H}\right), 2.99(1 \mathrm{H}, \mathrm{dd}$,

$\left.720 J 10.5,7.5, \mathrm{C}_{11 \mathrm{c}} \mathrm{H}\right), 4.15\left(1 \mathrm{H}, \mathrm{dd}, J 11.0,7.0, \mathrm{C}_{5 \mathrm{a}} \mathrm{H}\right), 4.33\left(1 \mathrm{H}, \mathrm{dt}, J 10.5,7.0, \mathrm{C}_{5} \mathrm{H}\right)$, $5.97\left(2 \mathrm{H}, A B, J 1.5, \mathrm{OCH}_{2} \mathrm{O}\right), 6.70\left(3 \mathrm{H}, \mathrm{m}, 3 \times \mathrm{C}_{\mathrm{ar}} \mathrm{H}\right) ; \delta_{\mathrm{C}}\left(\mathrm{CDCl}_{3}, 100 \mathrm{MHz}\right) 24.63$ (q), $27.54(\mathrm{q}), 30.70(\mathrm{t}), 32.98(\mathrm{t}), 35.40(\mathrm{q}), 42.77$ (d), $49.95(\mathrm{~d}), 57.84(\mathrm{t}), 68.59$ (d), 74.31 (d), 79.61 (d), 100.92 (t), 108.31 (d), 108.44 (s), 108.72 (d), 121.89 (d), 135.88 (s), 146.34 (s), 147.75 (s); $m / z\left(\mathrm{CI}^{+}\right) 332\left(\mathrm{MH}^{+}, 100\right)$; Found: $m / z \mathrm{MH}^{+}$, $725332.1867, \mathrm{C}_{19} \mathrm{H}_{26} \mathrm{NO}_{4}$, requires $332.1862(\Delta=+1.6)$.

\section{$\left(3 a R^{*}, 5 S^{*}, 6 R^{*}, 7 S^{*}, 7 \mathrm{a} R^{*}\right)-5,6-\mathrm{Di}-O$-isopropylidene-1- $N$-methyl-7-[3,4-} (methylenedioxy)-6-bromophenyl]-2,3,3a,4,5,6,7,7a-octahydroindole 27. To a solution of carbamate $( \pm)-\mathbf{2 5}(200 \mathrm{mg}, 0.46 \mathrm{mmol})$ and $\mathrm{K}_{2} \mathrm{CO}_{3}(69 \mathrm{mg}, 0.50 \mathrm{mmol})$ in $\mathrm{CH}_{2} \mathrm{Cl}_{2}(15 \mathrm{~mL})$ at $-78{ }^{\circ} \mathrm{C}$ was added $\mathrm{Br}_{2}$ dropwise. After stirring for $6 \mathrm{~h}$ at -78 $730{ }^{\circ} \mathrm{C}$, the reaction mixture was allowed to warm-up to RT over $14 \mathrm{~h} .{ }^{1} \mathrm{H}-\mathrm{NMR}$ of the crude suggested a partial deprotection of the acetonide. Hence, DMP $(61 \mu \mathrm{L}, 0.50$ $\mathrm{mmol}$ ) and $p$-TSA $(5 \mathrm{mg}, 0.03 \mathrm{mmol})$ were added at RT. After stirring for $40 \mathrm{~min}$, the reaction mixture was partitioned between $\mathrm{NaHCO}_{3}$ (sat. aq., $10 \mathrm{~mL}$ ) and $\mathrm{CH}_{2} \mathrm{Cl}_{2}$ $(10 \mathrm{~mL})$ and the organic phase was extracted with $\mathrm{CH}_{2} \mathrm{Cl}_{2}(10 \mathrm{~mL})$. The combined 735 organic phases were dried over $\mathrm{Na}_{2} \mathrm{SO}_{4}$, filtered and concentrated in vacuo. The residue was dissolved in degassed THF $(5 \mathrm{~mL})$ and $\mathrm{LiAlH}_{4}(52 \mathrm{mg}, 1.38 \mathrm{mmol})$ was added. The suspension was refluxed in the dark at $80{ }^{\circ} \mathrm{C}$ for $5 \mathrm{~h}$ and quenched with $\mathrm{MeOH}(2 \mathrm{~mL})$. The reaction mixture was partitioned between brine $(15 \mathrm{~mL})$ and $\mathrm{Et}_{2} \mathrm{O}(15 \mathrm{~mL})$ and the organic phase was extracted with $\mathrm{Et}_{2} \mathrm{O}(3 \times 15 \mathrm{~mL})$. The 740 combined organic phases were dried over $\mathrm{Na}_{2} \mathrm{SO}_{4}$, filtered and concentrated in vacuo. The residue was purified by $\mathrm{FC}\left(\mathrm{SiO}_{2} ; \mathrm{Et}_{2} \mathrm{O} /\right.$ petrol, $\left.5: 1 \rightarrow \mathrm{Et}_{2} \mathrm{O} 100 \%\right)$ to give bromo- $N$-methyl indoline $( \pm)-27$ as a yellow oil $(38 \mathrm{mg}, 21 \%) . \mathrm{R}_{f} 0.70$ $\left(\mathrm{Et}_{2} \mathrm{O} / \mathrm{MeOH}, 4: 1\right) ; v_{\max }$ (neat) $/ \mathrm{cm}^{-1} 983,1078\left(\mathrm{C}_{\mathrm{ar}}-\mathrm{Br}\right), 1187,1504,2875,2942 ; \delta_{\mathrm{H}}$ $\left(\mathrm{CDCl}_{3}, 400 \mathrm{MHz}\right) 1.29\left(3 \mathrm{H}, \mathrm{s}, \mathrm{CH}_{3}\right), 1.52\left(3 \mathrm{H}, \mathrm{s}, \mathrm{CH}_{3}\right), 1.71\left(1 \mathrm{H}, \mathrm{m}, \mathrm{C}_{4} H \mathrm{H}\right), 1.82$ $745\left(1 \mathrm{H}, \mathrm{m}, \mathrm{C}_{3} H \mathrm{H}\right), 1.90\left(3 \mathrm{H}, \mathrm{s}, \mathrm{NCH}_{3}\right), 1.96-2.14\left(3 \mathrm{H}, \mathrm{C}_{2} \mathrm{HH}, \mathrm{C}_{3} \mathrm{HH}, \mathrm{C}_{4} \mathrm{HH}\right), 2.15-2.22$ $\left(2 \mathrm{H}, \mathrm{C}_{2} H \mathrm{H} \& \mathrm{C}_{3 \mathrm{a}} \mathrm{H}\right), 2.99\left(1 \mathrm{H}, \mathrm{t}, J 11.0, \mathrm{C}_{11 \mathrm{~b}} \mathrm{H}\right), 3.54\left(1 \mathrm{H}, \mathrm{m}, \mathrm{C}_{11 \mathrm{c}} \mathrm{H}\right), 4.08(1 \mathrm{H}, \mathrm{dd}$, $\left.J 11.0,7.0, \mathrm{C}_{5 \mathrm{a}} \mathrm{H}\right), 4.38\left(1 \mathrm{H}, \mathrm{dt}, J 10.0,7.0, \mathrm{C}_{5} \mathrm{H}\right), 5.96(1 \mathrm{H}, \mathrm{d}, J 1.5, \mathrm{OCH} H \mathrm{O}), 6.00$ $(1 \mathrm{H}, \mathrm{d}, J 1.5, \mathrm{OCHHO}), 6.80\left(1 \mathrm{H}, \mathrm{s}, \mathrm{C}_{\mathrm{ar}} \mathrm{H}\right), 7.04\left(1 \mathrm{H}, \mathrm{s}, \mathrm{C}_{\mathrm{ar}} \mathrm{H}\right) ; \delta_{\mathrm{C}}\left(\mathrm{CDCl}_{3}, 100\right.$ MHz) 24.80 (q), 27.33 (q), 29.98 (t), 33.03 (t), 35.10 (q), 41.87 (d), 47.26 (d), 57.61 
$750(\mathrm{t}), 70.03(\mathrm{~d}), 74.21(\mathrm{~d}), 79.99$ (d), $101.72(\mathrm{t}), 107.56(\mathrm{~d}), 109.10(\mathrm{~s}), 112.85(\mathrm{~d})$, 116.78 (s), 135.10 (s), 146.73 (s), 147.59 (s); $/ z / z\left(\mathrm{CI}^{+}\right) 410\left[\mathrm{MH}^{+}\left({ }^{79} \mathrm{Br}\right), 100\right], 412$ $\left[\mathrm{MH}^{+}\left({ }^{81} \mathrm{Br}\right)\right.$, 97]; Found: $m / z\left[\mathrm{MH}^{+}\left({ }^{79} \mathrm{Br}\right)\right], 410.0973, \mathrm{C}_{19} \mathrm{H}_{25}{ }^{79} \mathrm{BrNO}_{4}$, requires $410.0967(\Delta=+1.5)$.

$\left(3 a R^{*}, 5 S^{*}, 6 R^{*}, 7 S^{*}, 7 \mathrm{a} R^{*}\right)$-5,6-Di- $O$-isopropylidene-1-(4-chlorobenzoyl)methyl755 7-[3,4-(methylenedioxy)phenyl]-2,3,3a,4,5,6,7,7a-octahydroindole 28 . To a solution of hydrindane $( \pm)-6(620 \mathrm{mg}, 1.96 \mathrm{mmol}), \mathrm{Et}_{3} \mathrm{~N}(1 \mathrm{~mL}, 7.82 \mathrm{mmol})$ and $4-$ DMAP (12 mg, $0.1 \mathrm{mmol})$ in $\mathrm{CH}_{2} \mathrm{Cl}_{2}(15 \mathrm{~mL})$ at $\mathrm{RT}$ was added 4-chlorobenzoyl chloride (441 mg, $2.52 \mathrm{mmol}$ ) dropwise. After stirring for $18 \mathrm{~h}$, the reaction mixture was partitioned between $\mathrm{NaHCO}_{3}$ (sat. aq., $10 \mathrm{~mL}$ ) and $\mathrm{CH}_{2} \mathrm{Cl}_{2}(5 \mathrm{~mL})$ and the 760 organic phase extracted. The combined organic phases were dried over $\mathrm{Na}_{2} \mathrm{SO}_{4}$, filtered and concentrated in vacuo. The residue was purified by $\mathrm{FC}\left(\mathrm{SiO}_{2} ; \mathrm{Et}_{2} \mathrm{O}\right)$ to give amide ( \pm )-28 as a white solid (545 $\mathrm{mg}, 61 \%)$ : Mp $186.4-188.3{ }^{\circ} \mathrm{C} . \mathrm{R}_{f} 0.45$ $\left(\mathrm{Et}_{2} \mathrm{O}\right) ; v_{\max } / \mathrm{cm}^{-1}$ (neat) $987,1081,1192,1516,1648(\mathrm{C}=\mathrm{O}), 2878,2939 ; \delta_{\mathrm{H}}\left(\mathrm{CDCl}_{3}\right.$, $400 \mathrm{MHz}) 1.30\left(3 \mathrm{H}, \mathrm{s}, \mathrm{CH}_{3}\right), 1.41\left(3 \mathrm{H}, \mathrm{s}, \mathrm{CH}_{3}\right), 1.55\left(1 \mathrm{H}, \mathrm{m}, \mathrm{C}_{4} \mathrm{HH}\right), 1.86-1.97(2 \mathrm{H}$,

$\left.765 \mathrm{C}_{4} \mathrm{HH} \& \mathrm{C}_{3} \mathrm{HH}\right), 2.03-2.18\left(2 \mathrm{H}, \mathrm{C}_{3} H \mathrm{H} \& \mathrm{C}_{2} \mathrm{H} H\right), 2.48\left(1 \mathrm{H}, \mathrm{m}, \mathrm{C}_{3 \mathrm{a}} \mathrm{H}\right), 2.74(1 \mathrm{H}, \mathrm{m}$, $\left.\mathrm{C}_{2} \mathrm{HH}\right), 3.54\left(2 \mathrm{H}, \mathrm{m}, \mathrm{C}_{11 \mathrm{~b}} \mathrm{H} \& \mathrm{C}_{11 \mathrm{c}} \mathrm{H}\right), 4.30\left(2 \mathrm{H}, \mathrm{m}, \mathrm{C}_{5} \mathrm{H} \& \mathrm{C}_{5 \mathrm{a}} \mathrm{H}\right), 5.85(1 \mathrm{H}, \mathrm{d}, J 1.5$, OCHHO), $5.90\left(1 \mathrm{H}, \mathrm{d}, J 1.5\right.$, OCHHO), $6.65\left(2.3 \mathrm{H}, \mathrm{m}, \mathrm{C}_{\mathrm{ar}} \mathrm{Hs}\right), 7.07(1.7 \mathrm{H}, \mathrm{m}$, $\left.\mathrm{C}_{\mathrm{ar}} \mathrm{Hs}\right), 7.26\left(3 \mathrm{H}, \mathrm{m}, 3 \times \mathrm{C}_{\mathrm{ar}} \mathrm{H}\right) ; \delta_{\mathrm{C}}\left(\mathrm{CDCl}_{3}, 100 \mathrm{MHz}\right) 24.91(\mathrm{q}), 27.84(\mathrm{q}), 29.70(\mathrm{t})$, $30.02(\mathrm{t}), 31.32(\mathrm{~d}), 34.36(\mathrm{t}), 47.24(2 \times \mathrm{d}), 73.08(\mathrm{~d}), 78.21(\mathrm{~d}), 100.92(\mathrm{t}), 108.47$ $770(2 \times \mathrm{d}), 108.70(\mathrm{~s}), 122.34(\mathrm{~d}), 128.22(2 \times \mathrm{d}), 128.73(2 \times \mathrm{d}), 132.74(\mathrm{~s}), 134.98(\mathrm{~s})$ $\left.135.81(\mathrm{~s}), 146.84(\mathrm{~s}), 147.81(\mathrm{~s}), 168.90(\mathrm{~s}) ; \mathrm{m} / z\left(\mathrm{Cl}^{+}\right) 456\left[\mathrm{MH}^{+}{ }^{35} \mathrm{Cl}\right), 100\right], 458$ [MH $\left.{ }^{+}{ }^{37} \mathrm{Cl}\right)$, 35]; Found: $\mathrm{m} / z\left[\mathrm{MH}^{+}\left({ }^{35} \mathrm{Cl}\right)\right], 456.1567, \mathrm{C}_{25} \mathrm{H}_{26}{ }^{35} \mathrm{ClNO}_{5}$, requires $456.1578(\Delta=+1.1 \mathrm{ppm})$; Calculated for $\mathrm{C}_{25} \mathrm{H}_{26} \mathrm{ClNO}_{5}: \mathrm{C}, 65.86 \% ; \mathrm{H}, 5.75 \% ; \mathrm{Cl}$ $7.78 \%$; N, 3.07\%; O $17.55 \%$, found C, $65.90 \% ; \mathrm{H}, 5.72 \% ; \mathrm{Cl}, 7.79 \%$;, $3.05 \%$; O

$77517.54 \%$. For a single crystal X-ray structure determination on this compound see ESI.

Crystal data for 28: $\mathrm{C}_{25} \mathrm{H}_{26} \mathrm{ClNO}_{5}, \mathrm{M}=455.92$, triclinic, $\mathrm{P}-1$ (no. 2), a $=6.0988(2)$, $\mathrm{b}=11.8551(4), \mathrm{c}=16.3205(5) \AA, \alpha=102.804(3), \beta=98.740(3), \gamma=102.130(3)^{\circ}, \mathrm{V}$ $=1100.31(6) \AA^{3}, \mathrm{Z}=2, \mathrm{D}_{\mathrm{c}}=1.376 \mathrm{~g} \mathrm{~cm}^{-3}, \mu(\mathrm{Cu}-\mathrm{K \alpha} \alpha)=1.854 \mathrm{~mm}^{-1}, \mathrm{~T}=298 \mathrm{~K}$, 780 colourless plates, Oxford Diffraction Xcalibur PX Ultra diffractometer; 4218 independent measured reflections $\left(R_{\text {int }}=0.0256\right), F^{2}$ refinement, $R_{1}(\mathrm{obs})=0.0368$, $\mathrm{wR}_{2}($ all $)=0.1087,3060$ independent observed absorption-corrected reflections $\left[\left|\mathrm{F}_{\mathrm{o}}\right|\right.$ $\left.>4 \sigma\left(\left|\mathrm{F}_{\mathrm{o}}\right|\right), 2 \theta_{\max }=143^{\circ}\right], 290$ parameters. CCDC 792819.

$\left(3 \mathrm{a} R^{*}, 5 S^{*}, 6 R^{*}, 7 S^{*}, 7 \mathrm{a} R^{*}\right)-5,6$-Di- $O$-isopropylidene-1-(4-chlorobenzyl)-7-[3,4785 (methylenedioxy)-6-oxomethylphenyl]-2,3,3a,4,5,6,7,7a-octahydroindole 29 and $\left(3 \mathrm{a} R^{*}, 5 S^{*}, 6 R^{*}, 7 S^{*}, 7 \mathrm{a} R^{*}\right)-5,6$-di- $O$-isopropylidene-1-(4-chlorobenzyl)-7-[3,4(methylenedioxy)phenyl]-2,3,3a,4,5,6,7,7a-octahydroindole 30. To a solution of amide ( \pm )-28 (164 mg, $0.36 \mathrm{mmol})$ and $\mathrm{K}_{2} \mathrm{CO}_{3}(102 \mathrm{mg}, 0.74 \mathrm{mmol})$ in $\mathrm{CH}_{2} \mathrm{Cl}_{2}(15$ $\mathrm{mL}$ ) at $-78{ }^{\circ} \mathrm{C}$ was added $\mathrm{Br}_{2}$ dropwise. After stirring for $6 \mathrm{~h}$ at $-78^{\circ} \mathrm{C}$, the reaction

790 mixture was allowed to warm to RT over $14 \mathrm{~h} .{ }^{1} \mathrm{H}-\mathrm{NMR}$ of the crude suggested a partial deprotection of the acetonide so 2,2-dimethoxypropane ( $54 \mu \mathrm{L}, 0.44 \mathrm{mmol})$ and $p$-TSA $(5 \mathrm{mg}, 0.03 \mathrm{mmol})$ were added at RT. After stirring for $40 \mathrm{~min}$, the reaction mixture was partitioned between $\mathrm{NaHCO}_{3}$ (sat. aq., $10 \mathrm{~mL}$ ) and $\mathrm{CH}_{2} \mathrm{Cl}_{2}(10$ $\mathrm{mL})$ and the organic phase was extracted with $\mathrm{CH}_{2} \mathrm{Cl}_{2}(10 \mathrm{~mL})$. The combined

795 organic phases were dried over $\mathrm{Na}_{2} \mathrm{SO}_{4}$, filtered and concentrated in vacuo. The residue $(150 \mathrm{mg}, 0.28 \mathrm{mmol})$ was dissolved in degassed THF $(5 \mathrm{~mL})$ and $\mathrm{LiAlH}_{4}$ $\left(55 \mathrm{mg}, 1.43 \mathrm{mmol}\right.$ ) was added. The suspension was refluxed in the dark at $80{ }^{\circ} \mathrm{C}$ for $5 \mathrm{~h}$ and quenched with $\mathrm{MeOH}(2 \mathrm{~mL})$. The reaction mixture was partitioned between brine $(15 \mathrm{~mL})$ and $\mathrm{Et}_{2} \mathrm{O}(15 \mathrm{~mL})$ and the organic phase was extracted with

$800 \mathrm{Et}_{2} \mathrm{O}(3 \times 15 \mathrm{~mL})$. The combined organic phases were dried over $\mathrm{Na}_{2} \mathrm{SO}_{4}$, filtered and concentrated in vacuo. The residue was purified by $\mathrm{FC}\left(\mathrm{SiO}_{2} ;\right.$ petrol/ $\left./ \mathrm{Et}_{2} \mathrm{O}, 3: 1\right)$ to give the intermediate aryl bromide as a white solid $(154 \mathrm{mg}, 0.29 \mathrm{mmol}, 80 \%): \mathrm{R}_{f}$ $0.55\left(\mathrm{Et}_{2} \mathrm{O} /\right.$ petrol, $\left.1: 2\right) ; \delta_{\mathrm{H}}\left(\mathrm{CDCl}_{3}, 400 \mathrm{MHz}\right) 1.27\left(3 \mathrm{H}, \mathrm{s}, \mathrm{CH}_{3}\right), 1.49(1 \mathrm{H}, \mathrm{m}$, $\left.\mathrm{C}_{4} H \mathrm{H}\right), 1.49\left(3 \mathrm{H}, \mathrm{s}, \mathrm{CH}_{3}\right), 1.80\left(1 \mathrm{H}\right.$, app td, $\left.J 13.6,10.2, \mathrm{C}_{4} \mathrm{HH}\right), 1.92(2 \mathrm{H}, \mathrm{m}$,

$\left.805 \mathrm{C}_{3} \mathrm{H}_{2}\right), 2.09\left(1 \mathrm{H}, \mathrm{ddd}, J 11.1,6.4,4.4, \mathrm{C}_{2} H \mathrm{H}\right), 2.21\left(1 \mathrm{H}, \mathrm{m}, \mathrm{C}_{3 \mathrm{a}} \mathrm{H}\right), 2.52(1 \mathrm{H}$, app t, $J$ $\left.11.1, \mathrm{C}_{2} \mathrm{H} H\right), 2.61\left(1 \mathrm{H}, \mathrm{d}, J 12.6 \mathrm{C}_{\mathrm{ar}} \mathrm{CH} H \mathrm{~N}\right), 2.75\left(1 \mathrm{H}, \mathrm{m}, \mathrm{C}_{11 \mathrm{c}} \mathrm{H}\right), 3.35(1 \mathrm{H}, \mathrm{d}, J$ 12.6, $\left.\mathrm{C}_{\mathrm{ar}} \mathrm{C} H \mathrm{HN}\right), 3.64\left(1 \mathrm{H}, \mathrm{t}, J 10.7, \mathrm{C}_{11 \mathrm{~b}} \mathrm{H}\right), 4.12\left(1 \mathrm{H}, \mathrm{dd}, J 10.7,7.1, \mathrm{C}_{5 \mathrm{a}} \mathrm{H}\right), 4.34$ $\left(1 \mathrm{H}\right.$, app dt, $\left.J 10.2,7.1, \mathrm{C}_{5} \mathrm{H}\right), 5.91(1 \mathrm{H}, A B, \mathrm{~J} 1.3, \mathrm{OCH} H \mathrm{O}), 5.95\left(1 \mathrm{H}, \mathrm{AB}_{\mathrm{q}}, 1.3\right.$, OCHHO), $6.76\left(1 \mathrm{H}, \mathrm{s}, \mathrm{C}_{\mathrm{ar}} \mathrm{H}\right), 6.97\left(1 \mathrm{H}, \mathrm{s}, \mathrm{C}_{\mathrm{ar}} \mathrm{H}\right), 7.11\left(2 \mathrm{H}, \mathrm{d}, J 8.4,2 \times \mathrm{C}_{\mathrm{ar}} \mathrm{H}\right), 7.17$

$810\left(2 \mathrm{H}, \mathrm{d}, J\right.$ 8.4, $\left.2 \times \mathrm{C}_{\mathrm{ar}} \mathrm{H}\right) ; \delta_{\mathrm{C}}\left(\mathrm{CDCl}_{3}, 100 \mathrm{MHz}\right) 24.72(\mathrm{q}), 27.35(\mathrm{q}), 30.36(\mathrm{t}), 32.88$ (t), $34.97(\mathrm{~d}), 47.70(\mathrm{~d}), 53.79(\mathrm{t}), 59.56(\mathrm{t}), 67.94(\mathrm{~d}), 74.11(\mathrm{~d}), 79.73(\mathrm{~d}), 101.77$ (t), 107.37 (d), $108.96(\mathrm{~s}), 112.91(\mathrm{~d}), 116.92(\mathrm{~s}), 128.11(2 \times \mathrm{d}), 130.21(2 \times \mathrm{d})$ $132.23(\mathrm{~s}), 134.87(\mathrm{~s}), 138.55(\mathrm{~s}), 146.80(\mathrm{~s}), 147.77(\mathrm{~s}) ; \mathrm{m} / \mathrm{z}\left(\mathrm{CI}^{+}\right) 520\left[\mathrm{MH}^{+}\left({ }^{79} \mathrm{Br}\right.\right.$, $\left.\left.{ }^{35} \mathrm{Cl}\right), 75\right], 522\left[\mathrm{MH}^{+}\left({ }^{79} \mathrm{Br},{ }^{37} \mathrm{Cl}\right),\left({ }^{81} \mathrm{Br},{ }^{35} \mathrm{Cl}\right), 100\right], 524\left[\mathrm{MH}^{+}\left({ }^{81} \mathrm{Br},{ }^{35} \mathrm{Cl}\right), 25\right]$; 815 Found: $m / z\left[\mathrm{MH}^{+}\left({ }^{79} \mathrm{Br},{ }^{35} \mathrm{Cl}\right)\right], 520.0884, \mathrm{C}_{25} \mathrm{H}_{28} \mathrm{NO}_{4}{ }^{35} \mathrm{Cl}^{79} \mathrm{Br}$, requires $520.0890(\Delta$ $=-1.2 \mathrm{ppm})$. The bromide was immediately dissolved in THF $(4 \mathrm{~mL})$ and cooled to $78^{\circ} \mathrm{C}$. To this solution was added tert-BuLi $(367 \mu \mathrm{L}, 1.5 \mathrm{M}$ in hexanes, $0.55 \mathrm{mmol})$ dropwise. After stirring for $1 \mathrm{~h}$ at $-78{ }^{\circ} \mathrm{C}$, DMF ( $341 \mu \mathrm{L}, 4.43 \mathrm{mmol}$ ) was added dropwise and the reaction mixture was allowed to warm to RT over $14 \mathrm{~h}$. The 820 resulting suspension was then partitioned between $\mathrm{NaHCO}_{3}$ (sat. aq., $10 \mathrm{~mL}$ ) and
$\mathrm{CH}_{2} \mathrm{Cl}_{2}(10 \mathrm{~mL})$ and the organic phase was extracted with $\mathrm{CH}_{2} \mathrm{Cl}_{2}(3 \times 10 \mathrm{~mL})$. The combined organic phases were dried over $\mathrm{Na}_{2} \mathrm{SO}_{4}$, filtered and concentrated in vacuo. The residue was purified by $\mathrm{FC}\left(\mathrm{SiO}_{2} ;\right.$ petrol//Et $\left.2 \mathrm{O}, 2: 1\right)$ to give:

Aldehyde $( \pm)-29$ as a white solid [ $86 \mathrm{mg}, 51 \%$ from amide $( \pm)-28$ ]: $\mathrm{Mp} 43.4-46.1{ }^{\circ} \mathrm{C}$ 825 (Et $\mathrm{E}_{2} \mathrm{O} /$ petrol); $\mathrm{R}_{f} 0.45$ (petrol/Et $\mathrm{Et}_{2} \mathrm{O}, 2: 1$ ); $v_{\max } / \mathrm{cm}^{-1}$ (neat) 983, 1078, 1187, 1206, $1697(\mathrm{C}=\mathrm{O}), 2875,2930 ; \delta_{\mathrm{H}}\left(\mathrm{CDCl}_{3}, 400 \mathrm{MHz}\right) 1.33\left(3 \mathrm{H}, \mathrm{s}, \mathrm{CH}_{3}\right), 1.44(3 \mathrm{H}, \mathrm{s}$, $\left.\mathrm{CH}_{3}\right), 1.55-1.71\left(2 \mathrm{H}, \mathrm{C}_{4} \mathrm{HH} \& \mathrm{C}_{3} H \mathrm{H}\right), 2.00-2.38\left(3 \mathrm{H}, \mathrm{C}_{3} H \mathrm{H}, \mathrm{C}_{2} \mathrm{HH} \& \mathrm{C}_{4} H \mathrm{H}\right), 2.34$ $\left(1 \mathrm{H}, \mathrm{m}, \mathrm{C}_{3 \mathrm{a}} \mathrm{H}\right), 2.69\left(1 \mathrm{H}, \operatorname{app} \mathrm{t}, J 10.0, \mathrm{C}_{2} H \mathrm{H}\right), 2.80\left(2 \mathrm{H}, \mathrm{m}, \mathrm{C}_{\mathrm{ar}} \mathrm{CH} H \mathrm{~N} \& \mathrm{C}_{11 \mathrm{c}} \mathrm{H}\right)$, $3.11\left(1 \mathrm{H}, \mathrm{d}, J 13.0, \mathrm{C}_{\mathrm{ar}} \mathrm{C} H \mathrm{HN}\right), 3.90\left(1 \mathrm{H}, \mathrm{t}, J 10.0, \mathrm{C}_{11 \mathrm{~b}} \mathrm{H}\right), 4.37\left(2 \mathrm{H}, \mathrm{m}, \mathrm{C}_{5} \mathrm{H} \&\right.$ $\left.830 \mathrm{C}_{5 \mathrm{a}} \mathrm{H}\right), 6.03(1 \mathrm{H}, A B, \mathrm{~J} 1.0, \mathrm{OCH} H \mathrm{O}), 6.07\left(1 \mathrm{H}, \mathrm{AB}_{\mathrm{q}}, 1.0, \mathrm{OCH} \mathrm{HO}\right), 6.91(2 \mathrm{H}, \mathrm{d}, J$ $\left.8.5,2 \times \mathrm{C}_{\mathrm{ar}} \mathrm{H}\right), 6.94\left(1 \mathrm{H}, \mathrm{s}, \mathrm{C}_{\mathrm{ar}} \mathrm{H}\right) 7.16\left(2 \mathrm{H}, \mathrm{d}, J 8.5,2 \times \mathrm{C}_{\mathrm{ar}} \mathrm{H}\right), 7.40\left(1 \mathrm{H}, \mathrm{s}, \mathrm{C}_{\mathrm{ar}} \mathrm{H}\right)$, $10.41(1 \mathrm{H}, \mathrm{s}, \mathrm{CHO}) ; \delta_{\mathrm{C}}\left(\mathrm{CDCl}_{3}, 100 \mathrm{MHz}\right) 24.29(\mathrm{q}), 27.18(\mathrm{q}), 31.24(\mathrm{t}), 32.59(\mathrm{t})$, 35.47 (d), 41.39 (d), $53.66(\mathrm{t}), 59.87(\mathrm{t}), 66.68(\mathrm{~d}), 74.02$ (d), 78.27 (d), 101.97 (t), 106.13 (d), 107.44 (d), 108.87 (s), $128.19(2 \times \mathrm{d}), 129.89(2 \times \mathrm{d}), 130.96(\mathrm{~s}), 132.44$ $835(\mathrm{~s}), 137.65(\mathrm{~s}), 141.60(\mathrm{~s}), 146.98(\mathrm{~s}), 152.72(\mathrm{~s}), 188.95$ (d); $\mathrm{m} / \mathrm{z}\left(\mathrm{CI}^{+}\right) 470$ $\left[\mathrm{MH}^{+}\left({ }^{35} \mathrm{Cl}\right), 100\right], 472\left[\mathrm{MH}^{+}\left({ }^{37} \mathrm{Cl}\right), 40\right]$; Found: $\mathrm{m} / \mathrm{z}\left[\mathrm{MH}^{+}\left({ }^{37} \mathrm{Cl}\right)\right], 470.1749$, $\mathrm{C}_{26} \mathrm{H}_{29} \mathrm{NO}_{5}{ }^{37} \mathrm{Cl}$, requires $470.1734(\Delta=+3.2 \mathrm{ppm})$; Calculated for $\mathrm{C}_{26} \mathrm{H}_{28} \mathrm{ClNO}_{5}$ : C, $66.45 \%$; $\mathrm{H}, 6.01 \%$; Cl, $7.54 \%$; N, 2.98\%; O, 17.02\%, found C, 66.47\%; H, 6.03\%; $\mathrm{Cl}, 7.52 \% ; \mathrm{N}, 2.97 \% ; \mathrm{O}, 17.01 \%$.

840 Reduced product $( \pm)-\mathbf{3 0}$ as a white solid [41 mg, $26 \%$ from amide $( \pm)-28]$ : Mp 34.2$38.6{ }^{\circ} \mathrm{C}\left(\mathrm{Et}_{2} \mathrm{O} /\right.$ petrol); $\mathrm{R}_{f} 0.35$ (petrol/ $\left.\mathrm{Et}_{2} \mathrm{O}, 2: 1\right) ; v_{\max } / \mathrm{cm}^{-1}$ (neat) 983, 1078, 1187, $1206,1505,2875,2930 ; \delta_{\mathrm{H}}\left(\mathrm{CDCl}_{3}, 400 \mathrm{MHz}\right) 1.33\left(3 \mathrm{H}, \mathrm{s}, \mathrm{CH}_{3}\right), 1.48\left(4 \mathrm{H}, \mathrm{s}, \mathrm{CH}_{3}\right.$ \& $\left.\mathrm{C}_{4} H \mathrm{H}\right), 1.68\left(1 \mathrm{H}, \mathrm{m}, \mathrm{C}_{3} \mathrm{HH}\right), 1.95-2.16\left(3 \mathrm{H}, \mathrm{C}_{3} H \mathrm{H}, \mathrm{C}_{2} \mathrm{HH} \& \mathrm{C}_{4} \mathrm{HH}\right), 2.26(1 \mathrm{H}$, $\left.\mathrm{m}, \mathrm{C}_{3 \mathrm{a}} \mathrm{H}\right), 2.64\left(1 \mathrm{H}, \mathrm{t}, J 10.0, \mathrm{C}_{2} \mathrm{HH}\right), 2.71\left(1 \mathrm{H}, \mathrm{d}, J 13.0, \mathrm{C}_{\mathrm{ar}} \mathrm{CH} H \mathrm{~N}\right), 2.82(2 \mathrm{H}, \mathrm{m}$, $\left.845 \mathrm{C}_{11 \mathrm{~b}} \mathrm{H}, \mathrm{C}_{11 \mathrm{c}} \mathrm{H}\right), 3.25\left(1 \mathrm{H}, \mathrm{d}, J\right.$ 13.0, $\left.\mathrm{C}_{\mathrm{ar}} \mathrm{C} H \mathrm{HN}\right), 4.26\left(1 \mathrm{H}, \mathrm{dd}, J 10.5,7.5, \mathrm{C}_{5 \mathrm{a}} \mathrm{H}\right), 4.34$ $\left(1 \mathrm{H}, \mathrm{m}, \mathrm{C}_{5} \mathrm{H}\right), 5.92(1 \mathrm{H}, \mathrm{d}, J 1.5, \mathrm{OCHHO}), 5.95(1 \mathrm{H}, \mathrm{d}, J 1.5, \mathrm{OCH} H \mathrm{O}), 6.77-6.85$ $\left(3 \mathrm{H}, 3 \times \mathrm{C}_{\mathrm{ar}} \mathrm{H}\right), 7.03\left(2 \mathrm{H}, \mathrm{d}, J 8.5, \mathrm{C}_{\mathrm{ar}} \mathrm{H}\right), 7.20\left(2 \mathrm{H}, \mathrm{d}, J 8.5, \mathrm{C}_{\mathrm{ar}} \mathrm{H}\right) ; \delta_{\mathrm{C}}\left(\mathrm{CDCl}_{3}, 100\right.$ MHz) 24.53 (q), 27.49 (q), 30.91 (t), 32.83 (t), 35.38 (d), 50.15 (d), 54.06 (t), 59.77 $(\mathrm{t}), 66.62(\mathrm{~d}), 74.25(\mathrm{~d}), 79.25(\mathrm{~d}), 100.95(\mathrm{t}), 108.38(\mathrm{~s}), 108.49$ (d), 108.62 (d), $850121.98(\mathrm{~d}), 128.25(2 \times \mathrm{d}), 129.56(2 \times \mathrm{d}), 132.21(\mathrm{~s}), 135.67(\mathrm{~s}), 138.60(\mathrm{~s}), 146.41$ (s), $147.88(\mathrm{~s}) ; \mathrm{m} / z\left(\mathrm{CI}^{+}\right) 442\left[\mathrm{MH}^{+}\left({ }^{35} \mathrm{Cl}\right), 100\right], 444\left[\mathrm{MH}^{+}\left({ }^{77} \mathrm{Cl}\right), 40\right]$; Found: $\mathrm{m} / z$ $\left[\mathrm{MH}^{+}\left({ }^{35} \mathrm{Cl}\right)\right], 442.1786, \mathrm{C}_{25} \mathrm{H}_{29} \mathrm{NO}_{4}{ }^{35} \mathrm{Cl}$, requires $442.1786(\Delta=+0.2 \mathrm{ppm})$; Calculated for $\mathrm{C}_{25} \mathrm{H}_{28} \mathrm{ClNO}_{4}$ : C, 67.94\%; H, 6.39\%; Cl, 8.02\%; N, 3.17\%; O, $14.48 \%$, found $\mathrm{C}, 67.89 \% ; \mathrm{H}, 6.41 \% ; \mathrm{Cl}, 8.01 \% ; \mathrm{N}, 3.15 \% ; \mathrm{O}, 14.54 \%$.

855 ( \pm )- 1 -(4-Chlorobenzyl)-1-desmethylclivonine 31. To a solution of aldehyde ( \pm )-29 $(20 \mathrm{mg}, 0.04 \mathrm{mmol})$ in THF $(3 \mathrm{~mL})$ at RT was added aq. $\mathrm{HCl}(0.5 \mathrm{~mL}, 2 \mathrm{M})$. After stirring for $3 \mathrm{~h}$ at $\mathrm{RT}$, the reaction mixture was freeze-dried and the residue was dissolved in toluene $\left(2 \mathrm{~mL} \text { ). Fetizon's reagent (i.e. } \mathrm{Ag}_{2} \mathrm{CO}_{3} \text {-Celite }{ }^{\circledR}\right)^{61}(327 \mathrm{mg}, 0.57$ mmol, $0.57 \mathrm{~g} / \mathrm{mol}$ ) was added and the reaction mixture heated for $1 \mathrm{~h}$ at $90{ }^{\circ} \mathrm{C}$. The 860 resulting black suspension was cooled to RT and filtered through Celite ${ }^{\circledR}$ eluting with EtOAc $(20 \mathrm{~mL})$. The filtrate and combined washings were concentrated in vacuo to yield a brown solid. This residue was purified by $\mathrm{FC}\left(\mathrm{SiO}_{2} ; \mathrm{Et}_{2} \mathrm{O}\right)$ and triturated with ice-cold $\mathrm{Et}_{2} \mathrm{O}(2 \mathrm{~mL})$ to give 1-(4-chlorobenzyl)-1desmethylclivonine $( \pm)-31$ as a white solid $(15 \mathrm{mg}, 82 \%)$ : Mp 247.8-250.2 ${ }^{\circ} \mathrm{C} ; \mathrm{R}_{f}$ $8650.55\left(\mathrm{Et}_{2} \mathrm{O}\right) ; v_{\max } / \mathrm{cm}^{-1}$ (neat) 984, 1078, 1187, 1206, 1505, $1735(\mathrm{C}=\mathrm{O}), 2875,2930$, $3205(\mathrm{OH}) ; \delta_{\mathrm{H}}\left(\mathrm{CDCl}_{3}, 400 \mathrm{MHz}\right) 1.87\left(1 \mathrm{H}, \mathrm{ddd}, J 15.0,6.5,4.0, \mathrm{C}_{4} \mathrm{HH}\right), 2.13(1 \mathrm{H}$, $\left.\mathrm{m}, \mathrm{C}_{3} \mathrm{HH}\right), 2.37\left(3 \mathrm{H}, \mathrm{m}, \mathrm{OH}, \mathrm{C}_{4} \mathrm{HH} \& \mathrm{C}_{3} \mathrm{HH}\right), 2.61\left(2 \mathrm{H}, \mathrm{m}, \mathrm{C}_{3 \mathrm{a}} \mathrm{H} \& \mathrm{C}_{2} \mathrm{H} H\right), 3.17$ $\left(2 \mathrm{H}, \mathrm{m}, \mathrm{C}_{2} \mathrm{HH} \& \mathrm{C}_{11 \mathrm{c}} \mathrm{H}\right), 3.39\left(1 \mathrm{H}, \mathrm{dd}, J 12.0,10.0, \mathrm{C}_{11 \mathrm{~b}} \mathrm{H}\right), 3.76(1 \mathrm{H}, \mathrm{d}, J 14.0$, $\left.\mathrm{C}_{\mathrm{ar}} \mathrm{C} H \mathrm{HN}\right), 4.03\left(1 \mathrm{H}, \mathrm{d}, J 14.0, \mathrm{C}_{\mathrm{ar}} \mathrm{CH} H \mathrm{~N}\right), 4.18\left(1 \mathrm{H}, \mathrm{dd}, J 12.0,2.5, \mathrm{C}_{5 \mathrm{a}} \mathrm{H}\right), 4.31$ $870\left(1 \mathrm{H}, \mathrm{dd}, J 6.5,2.5, \mathrm{C}_{5} \mathrm{H}\right), 6.05\left(2 \mathrm{H}, \mathrm{AB}_{\mathrm{q}}, J 1.5, \mathrm{OCH}_{2} \mathrm{O}\right), 7.29\left(2 \mathrm{H}, \mathrm{s}, 2 \times \mathrm{C}_{\mathrm{ar}} \mathrm{H}\right), 7.36$ $\left(2 \mathrm{H}, \mathrm{s}, 2 \times \mathrm{C}_{\mathrm{ar}} \mathrm{H}\right), 7.51\left(1 \mathrm{H}, \mathrm{s}, \mathrm{C}_{\mathrm{ar}} \mathrm{H}\right), 7.90\left(1 \mathrm{H}, \mathrm{s}, \mathrm{C}_{\mathrm{ar}} \mathrm{H}\right) ; \delta_{\mathrm{C}}\left(\mathrm{CDCl}_{3}, 100 \mathrm{MHz}\right) 28.59$ $(\mathrm{t}), 30.20(\mathrm{t}), 33.32(\mathrm{~d}), 34.18(\mathrm{~d}), 49.94(\mathrm{t}), 60.81(\mathrm{t}), 67.38(\mathrm{~d}), 68.90(\mathrm{~d}), 81.82(\mathrm{~d})$, $101.95(\mathrm{t}), 107.12$ (s), 109.36 (d), 118.67 (s), $128.25(2 \times \mathrm{d}), 129.68(2 \times \mathrm{d}), 132.21$ (s), 137.93 (s), 140.63 (s), 146.77 (s), 152.67 (s), 164.63 (s); m/z (CI $) 428[\mathrm{M}$ $\left.875 \mathrm{H}^{+}\left({ }^{35} \mathrm{Cl}\right), 100\right], 430 \quad\left[\mathrm{MH}^{+}\left({ }^{37} \mathrm{Cl}\right), 35\right]$; Found: $m / z \quad\left[\mathrm{MH}^{+}\left({ }^{35} \mathrm{Cl}\right)\right], 428.1256$, $\mathrm{C}_{23} \mathrm{H}_{22} \mathrm{NO}_{5}{ }^{35} \mathrm{Cl}$, requires $428.1265(\Delta=-2.0 \mathrm{ppm})$; Calculated for $\mathrm{C}_{23} \mathrm{H}_{22} \mathrm{ClNO}_{5}$ : $\mathrm{C}$, $64.56 \% ; \mathrm{H}, 5.18 \% ; \mathrm{Cl}, 8.29 \%$; N $3.27 \%$; O, $18.70 \%$, found $\mathrm{C}, 64.60 \%$; H, 5.19\%; $\mathrm{Cl}, 8.27 \%$; N, $3.26 \%$; O, $18.68 \%$.

\section{Acknowledgements}

880 The authors acknowledge the Swiss National Science Foundation, the EPSRC and GSK for financial support for this work. We thank Dr G. Whited (Genencor Inc.) for a generous gift of (+)-(2S,3S)-1-chloro-4,6-cyclohexadiene-2,3-diol.

\section{Notes and references}

Deceased March 15, 1996

$885 \div$ The absolute stereochemistry of $(+)-\alpha$-dihydrohippeastrine was secure from chemical correlation to dihydrolycorine hydrobromide, which had been subject 
to anomalous dispersion single crystal X-ray structure determination (see refs. ${ }^{16,17}$ ).

$\S \quad$ This compound is commercially available or can be prepared from 1,4-

$890 \quad$ cyclohexadiene ( 4 steps, $26 \%$ yield, see refs ${ }^{26-28}$ ) or from $m y o$-inositol ( 3 steps, $36 \%$ yield, see ref. ${ }^{29}$ ).

** Specifically, in the clivonine series H5 appears at $\delta 4.2-4.3 \mathrm{ppm}$ as a $d d d$ or $q$ $(J \sim 5 \mathrm{~Hz})$ and $\mathrm{H} 6$ at $\delta \sim 4.8 \mathrm{ppm}$ as a $d(J \sim 5 \mathrm{~Hz})$, whereas in the trianthine series the corresponding protons are downfield shifted: $\mathrm{H} 5$ at $\delta 4.5-4.6 \mathrm{ppm}$ and

$895 \quad \mathrm{H} 6$ at $\delta 4.9 \mathrm{ppm}$

i† Knight and Salter have proposed that allylic oxygenation favours retro-Cope elimination reactions but a strong conformational dependence for this has not been noted previously (se ref. ${ }^{40}$ ). That the retro-Cope elimination proceeds via a dissymmetric concerted pathway in which the hydroxylamine nitrogen has

900 some nucleophilic character and the alkene some electrophilic character is supported by computational studies by Tronchet (see ref. ${ }^{41}$ ) and by our own observations that these reactions are facilitated by electron withdrawing substituents on the alkene.

It Lactam 19 and amine 20 were explored as precursors to the corresponding 905 lactamol (hemiaminal) via 'half-reduction' (using $\mathrm{LiEt}_{3} \mathrm{BH}$ ) and oxidation (using Polonovski-Potier chemistry) respectively in our development of the biomimetic approach to clivonine (see ref. ${ }^{23}$ ).

$\S \S$ This oxidation appears to be difficult, Irie et al. obtained just a $10 \%$ yield of clivonine by oxidation of the same substance but lacking the acetonide protecting group using $\mathrm{MnO}_{2}$ (see refs ${ }^{20-22}$ ).

*** The broad peaks in the ${ }^{1} \mathrm{H}$ NMR spectra of carbamates 21,22 and 25 at RT became sharp when recorded at $\sim 330 \mathrm{~K}$. By contrast, the broad peaks in the ${ }^{1} \mathrm{H}$ NMR spectrum of amide $\mathbf{2 8}$ remained broad at $328 \mathrm{~K}$ suggesting higher barriers to aryl/aryl mutual rotation in this latter derivative.

915 1. Z. Jin, Nat. Prod. Rep., 2009, 26, 363-381 and previous reviews in this series

2. O. Hoshino, The Alkaloids, 1998, 51, 323-424.

3. S. F. Martin, The Alkaloids, 1987, 30, 251-376.

4. B. Weniger, L. Italiano, J.-P. Beck, J. Bastida, S. Bergoñon, C. Codina, A. Lobstein and R. Anton, Planta Med., 1995, 61, 77-89.

920 5. A. Evidente, Phytochemistry Reviews, 2009, 8, 449-459.

6. A. Evidente, A. S. Kireev, A. R. Jenkins, A. E. Romero, W. F. A. Steelant, S. V. Slambrouck and A. Kornienko, Planta Med., 2009, 75, 501-507.

7. G. Schmeda-Hirschmann, L. Astudillo, J. Bastida, F. Viladomat and C. Codina, Bol. Soc. Chil. Quim., 2000, 45, 1119.

9258 L. Szlávik, Á. Gyuris, J. Minárovits, P. Forgo, J. Molnár and J. Hohmann, Planta Med., 2004, 70, 871-873.

9. J. Renard-Nozaki, T. Kim, Y. Imakura, M. Kihara and S. Kobayashi, Res. Virol., 1989, 140, 115-128.

10. A. Evidente, A. Andolfi, A. H. Abou-Donia, S. M. Touema, H. M. Hammoda, E. Shawky 930 and A. Motta, Phytochemistry, 2004, 65, 2113-2118.

11. A. Numata, T. Takemura, H. Ohbayashi, T. Katsuno, K. Yamamoto, K. Sato and S Kobayashi, Chem. Pharm. Bull., 1983, 31, 2146.

12. J. Bastida, R. Lavilla and F. Viladomat, The Alkaloids, 2006, 63, 87-179.

13. C. K. Briggs, P. F. Highet, R. J. Highet and W. C. Wildman, J. Am. Chem. Soc., 1956, 78, 2835 289-2904.

14. B. Mehlis, Naturwissenschaften, 1965, 52, 33-34.

15. W. Dopke, M. Bienert, A. L. Burlingame, P. W. Jeffs and D. S. Farrier, Tetrahedron Letts., 1967, 451-457.

16. T. Kitagawa, S. Uyeo and N. Yokoyama, J. Chem. Soc., 1959, 3741-3751.

940 17. M. Shiro, T. Sato and H. Koyama, Chem. Ind., 1966, 1229.

18. P. W. Jeffs, J. F. Hansen, W. Dopke and M. Bienert, Tetrahedron, 1971, 27, 5065-5079.

19. H. K. Schnoes, D. H. Smith, A. L. Burlingame, P. W. Jeffs and W. Döpke, Tetrahedron, 1968, 24, 2825-2837.

20. H. Irie, Y. Nagai, K. Tamoto and H. Tanaka, J. Chem. Soc., Chem. Commun., 1973, 302945303

21. H. Tanaka, H. Irie, S. Babu, S. Uyeo, A. Kuno and Y. Ishiguro, J. Chem. Soc., Perkin Trans. I, 1979, 535-538

22. H. Tanaka, Y. Nagai, H. Irie, S. Uyeo and A. Kuno, J. Chem. Soc., Perkin Trans. 1, 1979, 874-878.

950 23. C. Giro-Manas, V. L. Paddock, C. G. Bochet, A. C. Spivey, A. J. P. White, I. Mann and W. Oppolzer, J. Am. Chem. Soc, 2010, 132, 5176-5178.

24. N. J. Cooper and D. W. Knight, Tetrahedron, 2004, 60, 243-269.

25. A. C. Spivey, Chimia, 2009, 63, 864-866

26. C. R. Johnson, P. A. Ple and J. P. Adams, J. Chem. Soc., Chem. Commun., 1991, 1006-1007.

955 27. Y. Sutbeyaz, H. Secen and M. Balci, J. Chem. Soc., Chem. Commun., 1988, 1330-1331.

28. N. C. Yang, M.-J. Chen and P. Chen, J. Am. Chem. Soc., 1984, 106, 7310-7315.

29. F. Fabris, E. Rosso, A. Paulon and O. De Lucchi, Tetrahedron Letts., 2006, 47, 4835-4837.

30. T. Hudlicky, R. L. Fan, T. Tsunoda, H. Luna, C. Andersen and J. D. Price, Isr. J. Chem. 1991, 31, 229-238.

960 31. K. Ramesh, M. S. Wolfe, Y. Lee, D. V. Velde and R. T. Borchardt, J. Org. Chem., 1992, 57, 5861-5868.

32. N. Kornblum and H. E. De La Mare, J. Am. Chem. Soc., 1951, 73, 880-881.

33. A. G. Griesbeck, T. Deufel, K. Peters, E.-M. Peters and H. G. von Schnering, J. Org. Chem, 1995, 60, 1952-1958.

965 34. A. C. Spivey, C. Giro-Manas and I. Mann, Chem. Commun., 2005, 4426-4428.

35. W. Oppolzer, A. C. Spivey and C. G. Bochet, J. Am. Chem. Soc., 1994, 116, 3139-3140.
36. E. Pretsch, P. Buhlmann and C. Affolter, Structure Determination of Organic Compounds Tables of Spectral Data, Springer, Berlin, 3rd edn., 2000.

37. L. O. Jeroncic, M. P. Cabal and S. J. Danishefsky, J. Org. Chem., 1991, 56, 387-395.

970 38. A. S. Cieplak, Chem. Rev, 1999, 99, 1265-1336.

39. For an excellent summary of potential stereoelectronic factors in this type of situation see: Fleming, I. 'Molecular Orbitals and Organic Chemical Reactions - Reference Edition', Wiley, Chichester, 2010, pp2246-2250.

40. D. W. Knight, Salter, R., Tetrahedron Letts., 1999, 40, 5915-5918.

975 41. I. Komaromi and J. M. J. Tronchet, J. Phys. Chem. A, 1997, 101, 3554-3560.

42. S. Mizukami, Tetrahedron, 1960, 11, 89-95.

43. K. Kotera, Y. Hamada and R. Nakane, Tetrahedron, 1968, 24, 759-770 and references therein.

44. R. D. Harken, C. P. Christensen and W. C. Wildman, J. Org. Chem., 1976, 41, 2450-2454.

$98045 . \quad$ H. A. Hageman, Org. React., 1953, 7, 198-262.

46. G. E. Keck and R. R. Webb, J. Am. Chem. Soc., 1981, 103, 3173-3177.

47. O. Hoshino, M. Ishizaki, K. Kamei, M. Taguchi, T. Nagao, K. Iwaoka, S. Sawaki, B. Umezawa and Y. Iitaka, Chem. Lett., 1991, 1365-1368.

48. H. U. Reissig and H. Lorey, Liebigs. Ann. Chem., 1986, 1914

985 49. M. G. Banwell, C. J. M. Cowden and R. W. Gable, J. Chem. Soc., Perkin Trans. 1, 1994, 3515.

50. M. G. Banwell, B. D. Bissett, S. Busato, C. J. Cowden, D. C. R. Hockless, J. W. Holman, R W. Read and A. W. Wu, J. Chem. Soc., Chem. Commun., 1995, 2551-2553.

51. J. D. Hobson and J. G. McCluskey, J. Chem.Soc. C: Organic, 1967, 2015-2017.

990 52. J. Campbell, J. Org. Chem., 1957, 22, 1259-1260.

53. R. A. Olofson, J. T. Martz, J. P. Senet, M. Piteau and T. Malfroot, J. Org. Chem., 1984, 49, 2081-2082.

54. H. Normant, T. Cuvigny and P. Savignac, Synthesis, 1975, 805

55. G. Gardillo, M. Orena, G. Porzi and S. Sandri, Synthesis, 1981, 793.

995 56. G. Gardillo, M. Orena and S. S., Tetrahedron Letts., 1976, 3985

57. V. Franzen and S. Otto, Berichte, 1961, 94, 1360

58. T. T. Tidwell, Synthesis, 1990, 1990, 857-870.

59. R. K. Boeckman, P. Shao and J. J. Mullins, Org. Synth., 2004, Coll. Vol. 10, 696.

60. H. Pinnick, W. E. Childers and B. Bal, Tetrahedron, 1981, 37, 2091.

1000 61. M. Fetizon, J. Org. Chem., 1971, 36, 1339-1341

62. L. E. Overman, C. Y. Hong and N. Kado, J. Am. Chem. Soc., 1993, 115, 11028-11029.

63. W. L. E. Armarego and C. L. L. Chai, Purification of Laboratory Chemicals, ButterworthHeinmann, New York, 5th Edition edn., 2003.

64. L. Zetta, G. Gatti and C. Fuganti, J. Chem. Soc., Perkin Trans. 2, 1973, 1180-1184.

1005 65. S. Kobayashi, H. Ishikawa, E. Sasakawa, M. Kihara, T. Shingu and A. Kato, Chem. Pharm. Bull., 1980, 28, 1827-1831.

66. J. Schreiber, H. Maag, N. Hashimoto and A. Eschenmoser, Angew. Chem. Int. Ed. Engl., $1971,10,330-331$

This journal is (C) The Royal Society of Chemistry [year] 
${ }_{1010}$ Graphical Abstract:

\section{Total Synthesis of the Amaryllidaceae Alkaloid Clivonine}

\section{Helmut Haning, ${ }^{\mathrm{a}, \mathrm{b}}$ Carles Giró Mañas, ${ }^{\mathrm{c}}$ Victoria L. Paddock, ${ }^{\mathrm{c}}$ Christian G. Bochet,${ }^{\mathrm{d}, \mathrm{b}}$ Andrew J. P. White, ${ }^{\mathrm{c}}$ Gerald Bernardinelli, ${ }^{\mathrm{b}}$ Inderjit Mann, ${ }^{\mathrm{e}}$ Wolfang Oppolzer, ${ }^{\dagger \mathrm{b}}$ and Alan C. Spivey ${ }^{* \mathrm{c}, \mathrm{b}}$}

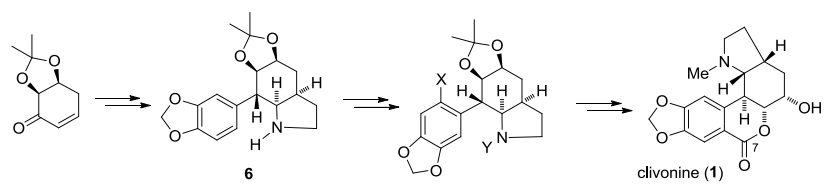

1015 Two syntheses of the Amaryllidaceae alkaloid clivonine (1) are described.

\footnotetext{
${ }^{\dagger}$ Deceased March 15, 1996.

The absolute stereochemistry of (+)- $\alpha$-dihydrohippeastrine was secure from chemical correlation to dihydrolycorine hydrobromide, which had been subject to anomalous dispersion single crystal X-ray structure determination (see refs.16. T. Kitagawa, S. Uyeo and N. Yokoyama, J. Chem. Soc., 1959, $3741-3751$. and 17. M. Shiro, T. Sato and H. Koyama, Chem. Ind., 1966, 1229.).

$\$$ This compound is commercially available or can be prepared from 1,4-cyclohexadiene (4 steps, $87 \%$ yield, see refs 26. Chem. Soc., Chem. Commun., 1991, 1006-1007, $27 . \quad$ Y. Sutbeyaz, H. Secen and M. Balci, J. Chem. Soc., Chem. Commun., 1988, 1330-1331, 28.
Yang, M.-J. Chen and P. Chen, J. Am. Chem. Soc., 1984, 106, 7310-7315.) or from myo-inositol (3 steps,36\% yield, see ref. 29. F. Fabris, E. Rosso, A. Paulon and O. De Lucchi, Tetrahedron Letts., 2006, 47, 4835-4837.).

Specifically, in the clivonine series $\mathrm{H} 5$ appears at $\delta 4.2-4.3 \mathrm{ppm}$ as a $d d d$ or $q(J \sim 5 \mathrm{~Hz})$ and $\mathrm{H} 6$ at $\delta \sim 4.8 \mathrm{ppm}$ as a $d(J \sim 5 \mathrm{~Hz})$, whereas in the trianthine series the corresponding protons are downfield shifted: $\mathrm{H} 5$ at $\delta 4.5-4.6 \mathrm{ppm}$ and $\mathrm{H} 6$ at $\delta 4.9 \mathrm{ppm}$.

ti Knight and Salter have proposed that allylic oxygenation favours retro-Cope elimination reactions but a strong conformational dependence for this has not been noted

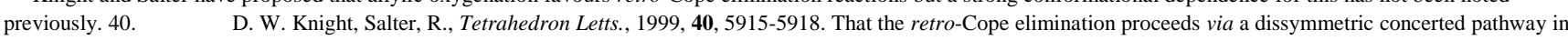
which the hydroxylamine nitrogen has some nucleophilic character and the alkene some electrophilic character is supported by computational studies by Tronchet (see ref.41.

I. Komaromi and J. M. J. Tronchet, J. Phys. Chem. A, 1997, 101, 3554-3560.) and by our own observations that these reactions are facilitated by electron withdrawing substituents on the alkene.

"Lactam 19 and amine 20 were explored as precursors to the corresponding lactamol (hemiaminal) via 'half-reduction' (using LiEt 3 BH) and oxidation (using PolonovskiPoitier chemistry) respectively in our development of the biomimetic approach to clivonine (see ref. 23 . C. Giro-Manas, V. L. Paddock, C. G. Bochet, A. C. Spivey, A. J. P. White, I. Mann and W. Oppolzer, J. Am. Chem. Soc, 2010, 132, 5176-5178.).

$\$$ This oxidation appears to be difficult, Irie et al. obtained just a $10 \%$ yield of clivonine by oxidation of the same substance but lacking the acetonide protecting group using $\mathrm{MnO}_{2}$ (see refs $20 . \quad$ H. Irie, Y. Nagai, K. Tamoto and H. Tanaka, J. Chem. Soc., Chem. Commun., 1973, 302-303, $21 . \quad$ H. Tanaka, H. Irie, S. Babu, S. Uyeo, A. Kuno and Y. Ishiguro, J. Chem. Soc., Perkin Trans. I, 1979, 535-538, $22 . \quad$ H. Tanaka, Y. Nagai, H. Irie, S. Uyeo and A. Kuno, J. Chem. Soc., Perkin Trans. 1, 1979, 874878.).

${ }^{* * * *}$ The broad peaks in the ${ }^{1} \mathrm{H}$ NMR spectra of carbamates $\mathbf{2 1 ,} 22$ and $\mathbf{2 5}$ at RT became sharp when recorded at $\sim 330 \mathrm{~K}$. By contrast, the broad peaks in the ${ }^{1} \mathrm{H}$ NMR spectrum of amide 27 remained unchanged at $328 \mathrm{~K}$ suggesting higher barriers to aryl/aryl mutual rotation in this latter derivative.
} 